\title{
Breaking ties in collective decision-making
}

\author{
Daniela Bubboloni ${ }^{1}\left[\right.$ [D $\cdot$ Michele Gori ${ }^{2}$
}

Received: 5 February 2020 / Accepted: 13 July 2020 / Published online: 26 August 2020

(c) The Author(s) 2020

\begin{abstract}
Many classic social preference (multiwinner social choice) correspondences are resolute only when two alternatives and an odd number of individuals are considered. Thus, they generally admit several resolute refinements, each of them naturally interpreted as a tie-breaking rule. A tie-breaking rule is compulsory every time a single final decision is needed. Unfortunately, using a tie-breaking rule on some social preference (multiwinner social choice) correspondence can dramatically compromise its properties. In particular, very often, the arithmetic relation between the number of alternatives and the number of voters does not allow to maintain both anonymity and neutrality. In those cases, the only possibility is to look at suitable different forms of symmetry that are coherent with the decision context. We find out conditions which make a social preference (multiwinner social choice) correspondence admit a resolute refinement fulfilling some weak versions of the anonymity and neutrality principles. We also clear when it is possible to obtain, for those resolute refinements, the reversal symmetry (immunity to the reversal bias). The theory we develop turns out to be useful in many common applicative contexts and allows to explicitly construct those refinements.
\end{abstract}

Keywords Social preference correspondence $\cdot$ Multiwinner social choice correspondence $\cdot$ Resoluteness $\cdot$ Anonymity $\cdot$ Neutrality $\cdot$ Tie-breaking rule

\section{JEL Classification D71}

Daniela Bubboloni was supported by GNSAGA of INdAM.

Michele Gori

michele.gori@unifi.it

Daniela Bubboloni

daniela.bubboloni@unifi.it

1 Dipartimento di Matematica e Informatica “Ulisse Dini”, Università degli Studi di Firenze, viale Morgagni 67/a, 50134 Florence, Italy

2 Dipartimento di Scienze per l'Economia e l'Impresa, Università degli Studi di Firenze, via delle Pandette 9, 50127 Florence, Italy 


\section{Introduction}

Consider a committee having $h \geq 2$ members whose purpose is to determine a ranking of $n \geq 2$ alternatives and assume that committee members are supposed to express their preferences via a ranking of the alternatives. A preference profile is a list of individual preferences, one for each committee member. A social preference correspondence (SPC) is a procedure which associates with any preference profiles a family of rankings of the alternatives to be interpreted as the family of the social preferences which better fit the individual preferences. A $k$-multiwinner social choice correspondence $(k$-SCC) is instead a procedure which associates with any preference profile a family of sets of $k$ alternatives to be interpreted as the family of all the sets of $k$ alternatives that can be considered the best $k$ alternatives for the society. While the concept of SPC is classic, the one of $k$-SCC, which extends the well-established single-winner framework (corresponding to the case $k=1$ ), is more recent. Indeed, it has been explored for about thirty years, but only for some years it has been getting a new attention. We refer to the recent papers by Elkind et al. (2017) and Faliszewski et al. (2017) for general information on that topic.

Many SPCs and $k$-SCCs have been proposed and studied in the literature. Most of them satisfy two requirements which are considered strongly desirable by social choice theorists, namely anonymity and neutrality. A SPC ( $k$-SCCs) is said anonymous if the identities of individuals are irrelevant to determine the social outcome, that is, it selects the same social outcome for any pair of preference profiles such that we get one from the other by permuting individual names; neutral if alternatives are equally treated, that is, for every pair of preference profiles such that we get one from the other by permuting alternative names, the social outcomes associated with them coincide up to the considered permutation.

Since in many cases collective decision processes are required to select a unique outcome, an important role in social choice theory is played by resolute SPCs and $k$-SCCs, namely those SPCs and $k$-SCCs associating a single ranking or a single set of $k$ alternatives with any preference profile. Unfortunately, classic SPCs and $k$-SCCs are not resolute in general. As a consequence, if the members of a committee agree to use one of the classic SPCs to aggregate their preferences and a unique outcome is needed, then they also need to find an agreement on which tie-breaking rule to use when two or more social rankings are selected. Similarly, if they agree to select the best $k$ alternatives for the society via a classic $k$-SCC and a unique outcome is needed, then they also need to decide which tie-breaking rule to use when two or more sets of $k$ alternatives are selected.

The choice of a tie-breaking rule usually has a deep impact on the properties of the final decision rule. Consider, for instance, two special tie-breaking rules often used in the single-winner framework. The first one, proposed by Moulin (1988), is based on a tie-breaking agenda, that is, an exogenously given ranking of the alternatives: In ambiguity case, it is chosen the alternative of the social outcome which is best ranked in the agenda. The second one is instead based on the preferences of one of the individuals appointed as tie-breaker: In ambiguity case, it is chosen the alternative of the social outcome which is best ranked by the tie-breaker. Of course, the resolute refinements built through a tie-breaking agenda fail to be neutral while those built 
through a tie-breaker fail to be anonymous. Our work allows to cast these two special types of tie-breaking rules within a more general theory (Sect. 8). ${ }^{1}$ We firmly believe that a reflection on the concept of tie-breaking rule is mandatory for social choice scientists in order to control the effective properties of the various resolute SPCs and $k$-SPCs in use and of those which could be used in the future. Indeed, it is clearly useless to choose a SPC or a $k$-SCC full of desirable properties and spoil a large part of them through an inappropriate tie-breaking rule.

On the same line of thought, Jeong and Ju (2017) observe that it is unfortunate that tie-breaking rules are not incorporated in the definitions of the classic 1-SCCs. In the context of two alternatives and allowing individuals to express also indifference between them, they discuss anonymous but non-neutral tie-breaking rules for the simple majority and the majority with quorum. In the same framework, but with profiles of variable length, McMorris et al. (2020) discuss many variations of the simple majority and, among them, they consider some of its resolute versions obtained through non-anonymous tie-breaking rules. Their scope is to characterize those variations in terms of weak versions of classic axioms and focusing on consistency. Thus, those authors implicitly treat also the properties which can derive by the use of different tie-breaking rules.

In order to better explain our results and simplify the exposition, let us focus now on SPCs. The concept of tie-breaking rule can be naturally formalized in terms of refinement of a SPC. Let $C$ and $C^{\prime}$ be two SPCs. We say that $C^{\prime}$ is a refinement of $C$ if, for every preference profile, the set of social preferences selected by $C^{\prime}$ is a subset of the set of social preferences selected by $C$. Thus, refinements of $C$ can be thought as methods to reduce the ambiguity in the choice made by $C$. In particular, resolute refinements of $C$ completely eliminate the ambiguity leading to a unique social outcome, so that they can be identified with the possible tie-breaking rules for $C$. Of course, if $C$ is not resolute, it admits many resolute refinements. Thus, an interesting issue to address is to understand whether it is possible to find resolute refinements of $C$ which satisfy suitable properties. In particular, since it is immediate to understand that resolute refinements of even anonymous and neutral SPCs are not generally anonymous and neutral, one may wonder whether anonymous and neutral resolute refinements of a given SPC do exist. Unfortunately, as proved by Bubboloni and Gori (2014, Theorem $5)$, the existence of an anonymous, neutral and resolute SPC is equivalent to the strong arithmetical condition $\operatorname{gcd}(h, n !)=1$ which is rarely satisfied in practical situations. As a consequence, given a SPC, we cannot in general get any anonymous and neutral resolute refinement of it. The condition $\operatorname{gcd}(h, n !)=1$ was first introduced by Moulin (1983, Theorem 1, p.25) as a necessary and sufficient condition for the existence of anonymous, neutral and efficient social choice functions; Bubboloni and Gori (2014, Theorem 15) generalize Moulin's result considering in addition the qualified majority principle; Campbell and Kelly (2015) show that if $n>h$ (so that $\operatorname{gcd}(h, n !) \neq 1)$ and

\footnotetext{
1 Some remarks about the tie-breaking agenda and the role of the tie-breaker are proposed in Doğan and Giritligil (2015) and Bubboloni and Gori (2016b), where the problem of finding resolute refinements of 1 - SCCs is considered.
} 
anonymous, neutral and resolute social choice functions exist, then those functions not only are inefficient but exhibit even more undesirable behaviors. ${ }^{2}$

Fortunately, in many concrete cases, anonymity and neutrality are too strong requirements. That happens, for instance, for those committees having a president who is appointed to be more influential than the other committee members or when a committee evaluates job candidates giving the female candidates an advantage over the male ones. Indeed, in such situations, individual and alternative names are not immaterial. For such a reason, we propose generalized versions of anonymity and neutrality which can take into account possible distinctions among individuals and among alternatives. Let $S_{h}$ be the group of permutations of the set $H=\{1, \ldots, h\}$ of committee member names and $S_{n}$ be the group of permutations of the set $N=\{1, \ldots, n\}$ of alternative names. Given a subgroup $V$ of $S_{h}$, we say that a SPC is $V$-anonymous if it selects the same social outcome for any pair of preference profiles such that we get one from the other by permuting individual names according to a permutation in $V$; given a subgroup $W$ of $S_{n}$, we say that a SPC is $W$-neutral if for every pair of preference profiles such that we get one from the other by permuting alternative names according to a permutation in $W$, it associates with them social outcomes which coincide up to the considered permutation. The special groups of permutations $V$ and $W$ need to be identified on the basis of the specific features of the situation at hand. Of course, $S_{h^{-}}$ anonymity corresponds to anonymity and $S_{n}$-neutrality corresponds to neutrality. As a consequence, each anonymous (neutral) SPC is $V$-anonymous ( $W$-neutral) for every $V(W)$. It is worth noting that, depending on the particular structure of $V$ and $W$, it is possible to get $V$-anonymous, $W$-neutral and resolute SPCs even when no anonymous, neutral and resolute SPC exists. ${ }^{3}$ That implies that, in a situation where suitable weak versions of anonymity and neutrality are sensible, there may be room for finding a resolute SPC satisfying such versions of anonymity and neutrality by refining a given anonymous and neutral SPC.

In this paper, we propose conditions on $V$ and $W$ which are necessary and sufficient to make a $V$-anonymous and $W$-neutral SPC admit a $V$-anonymous and $W$-neutral resolute refinement. Those conditions are summarized by an arithmetical relation between the size $|W|$ of $W$ and a special number $\gamma(V)$ associated with $V$ (defined in (12)). Namely, given a $V$-anonymous and $W$-neutral SPC $C$ (like, for instance, the Borda, the Copeland, the Minimax and the Kemeny SPCs which are anonymous and neutral and then $V$-anonymous and $W$-neutral for every choice of $V$ and $W$ ), we have that $C$ admits a $V$-anonymous and $W$-neutral resolute refinement if and only if ${ }^{4}$

$$
\operatorname{gcd}(\gamma(V),|W|)=1
$$

\footnotetext{
2 The existence of anonymous, neutral and resolute SPCs satisfying further properties is also studied by Bubboloni and Gori (2015), who focus on the majority principle, and Doğan and Giritligil (2015), who instead focus on monotonicity properties.

3 For instance, in the trivial case where $V$ and $W$ are singletons whose unique element is the identity permutation, every resolute SPC is $V$-anonymous and $W$-neutral.

4 Throughout the paper, given integers $x_{1}, \ldots, x_{s}$, for some $s \in \mathbb{N}$, we denote by $\operatorname{gcd}\left(x_{1}, \ldots, x_{s}\right)$ their greatest common divisor and by $\mathrm{lcm}\left(x_{1}, \ldots, x_{s}\right)$ their least common multiple.
} 
(Theorem 17). The computation of the numbers $\gamma(V)$ and $|W|$ is, in principle, hard. Fortunately, it becomes easy for many subgroups of interest in the applications (see Sect. 8). For example, consider a situation where individuals can be divided into disjoint subcommittees, say $H_{1}, \ldots, H_{r}$, where individuals have the same decision power and that alternatives can be divided into disjoint subclasses, say $N_{1}, \ldots, N_{s}$, where alternatives have the same exogenous importance. Assume first that such subcommittees (subclasses) can be ranked in such a way that, for every pair of subcommittees (subclasses), each individual (alternative) belonging to the subcommittee (subclass) having higher rank is more influential (more important) than any individual (alternative) belonging to the other subcommittee (subclass) ${ }^{5}$ In this case, it is natural to require a collective decision process not to distinguish among individuals (alternatives) belonging to the same subcommittee (subclass). Thus, two groups $V$ and $W$ naturally arise: $V$ is given by those permutations of individual names mapping every $H_{i}$ into itself; $W$ is given by those permutations of alternative names mapping every $N_{j}$ into itself. Clearly $|W|=\prod_{j=1}^{s}\left|N_{j}\right|$ ! and we show that

$$
\gamma(V)=\operatorname{gcd}\left(\left|H_{1}\right|, \ldots,\left|H_{r}\right|\right)
$$

${ }^{6}$ so that condition (1) becomes ${ }^{7}$

$$
\operatorname{gcd}\left(\left|H_{1}\right|, \ldots,\left|H_{r}\right|, \prod_{j=1}^{s}\left|N_{j}\right| !\right)=1 .
$$

Assume now that subclasses can be ranked as before while no ranking of subcommittees is available. However, it is known that subcommittees having the same size must have the same impact in the final decision. This particular situation leads to consider a collective decision process which does not distinguish among individuals (alternatives) belonging to the same subcommittee (subclass) and also does not distinguish among subcommittees having the same size. Thus, $V$ is now given by those permutations of individual names mapping every $H_{i}$ into one of the subcommittees having its same size (possibly itself), while $W$ is the same as before. We prove then that

$$
\gamma(V)=\operatorname{gcd}\left(\left|H_{1}\right| t_{1}, \ldots,\left|H_{r}\right| t_{r}\right),
$$

where $t_{i}$ counts the number of subcommittees of size $\left|H_{i}\right|$, so that condition (1) becomes

$$
\operatorname{gcd}\left(\left|H_{1}\right| t_{1}, \ldots,\left|H_{r}\right| t_{r}, \prod_{j=1}^{s}\left|N_{j}\right| !\right)=1
$$

\footnotetext{
5 The presence of a president and the case of gender discrimination previously mentioned are special instances.

6 We stress that equality in (2) along with the described results about $k$-SCCs (Theorems 20 and 21) imply as an immediate consequence the main result in Bubboloni and Gori (2016b), namely Theorem 8 therein.

7 Note that anonymity corresponds to have $H$ as a unique subcommittee and neutrality corresponds to have $N$ as a unique subclass. In this special case, (3) reduces to the already commented condition $\operatorname{gcd}(h, n !)=1$.
} 
We further deepen the analysis of the resolute refinements of a given SPC by considering the property of reversal symmetry, a property first introduced by Saari (1994). Recall that the reversal of a ranking of alternatives is the ranking obtained by making the best alternative the worst, the second best alternative the second worst, and so on, and that a SPC is said reversal symmetric if, for any pair of preference profiles such that one is obtained by the other by reversing each individual preference, it associates with one of them a set of social preferences if and only if it associates with the other one the set of their reversal. We prove that the condition

$$
\operatorname{gcd}(\gamma(V), \operatorname{lcm}(|W|, 2))=1
$$

along with other technical conditions, is necessary and sufficient to make any $V$ anonymous, $W$-neutral and reversal symmetric SPC admit a $V$-anonymous, $W$-neutral and reversal symmetric resolute refinement (Theorem 18). In particular, from that result, it is easily deduced that the Borda and the Copeland SPCs admit a $V$-anonymous, $W$-neutral and reversal symmetric resolute refinement if and only if (4) holds true.

Along with the described analysis of SPCs, we also study the problem to determine whether a $k$-SCC admits $V$-anonymous and $W$-neutral resolute refinements ${ }^{8}$ and whether some of them are immune to the reversal bias too. Recall that a $k$-SCC is immune to the reversal bias if it never associates the same set of $k$ alternatives with a preference profile and its reversal (see Saari and Barney 2003; Bubboloni and Gori 2016a). We prove that if (1) holds true, then any $V$-anonymous and $W$-neutral $k$-SCC admits a $V$-anonymous and $W$-neutral resolute refinement (Theorem 20). Assuming (4), we also find out conditions to make every $V$-anonymous, $W$-neutral and immune to the reversal bias $k$-SCC admit a resolute refinement having the same properties (Theorem 21). Remarkably, that analysis put in evidence the role played by the number $k$ of selected alternatives with respect to the number $n$ of alternatives, pointing out that the existence of an immune to the reversal bias resolute refinement is guaranteed when the pair $(n, k)$ belongs to a very specific set.

We emphasize that the methods developed in the paper allow to deal with situations in which forms of symmetry conceptually different from $V$-anonymity and $W$-neutrality are required. The idea is to give room to any possible mixture of the anonymity and neutrality principles through the consideration of generic subgroups $U$ of $S_{h} \times S_{n}$ and the corresponding definition of $U$-symmetry and $U$-consistency given for SPCs and $k$-SCCs (see (7), (8), (9)). That is done not in view of a mere and vacuous generalization, but in order to capture every possible decision scenario. As a concrete application, we examine, in detail, the case of a committee facing the problem of electing a subcommittee of size $k$ among $n$ members of the committee itself who run as candidates. In this particular situation, a collective decision process should make no distinction among individuals who are candidates and among individuals who are not candidates and, at the same time, it must carefully take into account the fact that the alternatives to choose from are now a subset of the set of individuals. In Sect. 8.3, we prove that if $\operatorname{gcd}(h, n)=1$, then any anonymous and neutral $k$-SCC admits a resolute refinement consistent with the above described requirements.

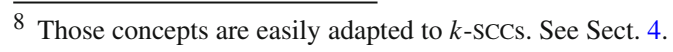


Note that some contributions dealing with weak versions of anonymity and neutrality are present in the literature. Assuming there are only two alternatives, Perry and Powers (2008) calculate the number of resolute SPCs that are anonymous and neutral and the number of SPCs satisfying a weak version of anonymity (that is, all individuals but one are anonymous) and neutrality; Powers (2010) shows that a SPC satisfies the previously described weak anonymity, neutrality and Maskin monotonicity if and only if it is close to SPC obeying an absolute qualified majority; Quesada (2013) identifies seven axioms (among which there are weak versions of anonymity and neutrality) characterizing the rules that are either the relative majority rule or the relative majority rule where a given individual can break the ties; Campbell and Kelly (2011, 2013) show that the relative majority is implied both by a suitable weak version of anonymity, neutrality and monotonicity, as well as by limited neutrality, anonymity and monotonicity. In the general case for the number of alternatives, some observations about different levels of anonymity and neutrality can be found in the paper by Kelly (1991). Bubboloni and Gori (2016b) consider, only for 1- SCCs, weak versions of anonymity and neutrality, determined by partitions of individuals and alternatives. More recently, a wide literature about $k$-SCCs which are representation-focused on some attributes of the alternatives (sex, age, profession, ethnicity) has been growing. See, for instance, Lang and Skowron (2016), Bredereck et al. (2018), Celis et al. (2018).

The techniques used in our paper are based on an algebraic approach focused on the theory of finite permutation groups and the notion of action of a group on a set. That approach was initially used by Eğecioğlu (2009) and Eğecioğlu and Giritligil (2013) for analyzing the set of preference profiles and later developed by Bubboloni and Gori $(2014,2015,2016 b)$ to describe, in a unified framework, profiles and functions on them satisfying some symmetry properties. ${ }^{9}$

We emphasize that, generalizing ideas and results in Bubboloni and Gori (2015, $2016 b$ ) for managing the resolute refinements of SPCs and $k$-SCC is in no way a trivial adaptation of the results in there. On the contrary, some proofs require complex and tricky arguments and, in particular, a deep consideration of the concept of regular group, first introduced in Bubboloni and Gori (2015), is needed.

The paper is organized as follows. In Sects. 2, 3 and 4 we give the definitions, the notation and the basic results needed to understand the model. In Sect. 5, the main results are stated and developed. Section 6 is devoted to a careful analysis of the concept of regular group. Section 7 explores $V$-anonymity and $W$-neutrality as an application of the main results of the general theory. Section 8 is about some concrete applications of $V$-anonymity and $W$-neutrality, as described in this introduction. Finally, appendices $\mathrm{A}$ and $\mathrm{B}$ contain the proofs of the main results.

\footnotetext{
${ }^{9}$ It is worth mentioning that Kelly (1991) discusses the role of symmetry in the arrovian framework through suitable subgroups of the symmetric group. Within the topological approach to social choices developed by Chichilnisky (1980), several algebraic concepts, in particular that of symmetric group, are crucial for proving many results. The algebraic approach also appears in Doğan and Giritligil (2015).
} 


\section{Preliminaries}

Throughout the paper, $\mathbb{N}$ denotes the set of positive integers and we set $\mathbb{N}_{0}=\mathbb{N} \cup\{0\}$. For $k \in \mathbb{N}$, the set $\{1, \ldots, k\}$ is denoted by $\llbracket k \rrbracket$. Let $X$ be a finite set. We denote by $|X|$ the size of $X$. A subset of $X$ of size $k$ is called a $k$-subset of $X$. We denote by $\mathbb{P}(X)$ the set of the subsets of $X$ and by $\mathbb{P}_{k}(X)$ the set of the $k$-subsets of $X$.

A relation on $X$ is a subset of $X^{2}$. The set of the relations on $X$ is denoted by $\mathbf{R}(X)$. Fix $R \in \mathbf{R}(X)$. Given $x, y \in X$, we usually write $x \succeq_{R} y$ instead of $(x, y) \in R$ and $x \succ_{R} y$ instead of $(x, y) \in R$ and $(y, x) \notin R$. We say that $R$ is complete if, for every $x, y \in X, x \succeq_{R} y$ or $y \succeq_{R} x$; reflexive if, for every $x \in X, x \succeq_{R} x$; irreflexive if, for every $x \in X, x \nsucceq_{R} x$; antisymmetric if, for every $x, y \in X, x \succeq_{R} y$ and $y \succeq_{R} x$ imply $x=y$; asymmetric if, for every $x, y \in X, x \succeq_{R} y$ implies $y \nsucceq_{R} x$; transitive if, for every $x, y, z \in X, x \succeq_{R} y$ and $y \succeq_{R} z$ imply $x \succeq_{R} z$; acyclic if, for every sequence $x_{1}, \ldots, x_{s}$ of $s \geq 2$ distinct elements of $X$ such that $x_{i} \succeq_{R} x_{i+1}$ for all $i \in \llbracket s-1 \rrbracket$, we have that $x_{S} \nsucceq_{R} x_{1}$. It is well known that if $R$ is transitive and irreflexive (antisymmetric, asymmetric), then $R$ is acyclic. Complete and transitive relations on $X$ are called orders on $X$. The set of orders on $X$ is denoted by $\mathbf{O}(X)$. Complete, transitive and antisymmetric relations on $X$ are called linear orders on $X$. The set of linear orders on $X$ is denoted by $\mathbf{L}(X)$. Given $R^{\prime} \in \mathbf{R}(X)$, if $R^{\prime} \subseteq R$ we say that $R^{\prime}$ refines $R$.

In the paper, we make use of basic finite group theory and use standard notation. Our general reference is Jacobson (1974). For completeness, we give here the main notions and notation we are going to use. Let $G$ be a finite group. Given $g \in G$, we denote by $|g|$ the order of $g$. If $U$ is a subgroup of $G$, we use the notation $U \leq G$. Given $g, v \in G$ and $U \leq G$, the conjugate of $g$ by $v$ is $g^{v}=v g v^{-1}$ and the conjugate of $U$ by $v$ is the subgroup $U^{v}=\left\{g^{v} \in G: g \in U\right\}$. We say that $g_{1}, g_{2} \in G$ are conjugate if there exists $v \in G$ such that $g_{2}=g_{1}^{v}$. The group constituted only by the neutral element is called the trivial group.

Let $n \in \mathbb{N}$. We denote by $S_{n}$ the group of the bijective functions from $\llbracket n \rrbracket$ to itself with product defined, for every $\sigma_{1}, \sigma_{2} \in S_{n}$, by the composition ${ }^{10} \sigma_{1} \sigma_{2} \in S_{n}$. The neutral element of $S_{n}$ is given by the identity function on $\llbracket n \rrbracket$, denoted by id. $S_{n}$ is called the symmetric group on $\llbracket n \rrbracket$, and its elements are called permutations. A permutation $\sigma \in S_{n} \backslash\{\mathrm{id}\}$ is the product of disjoint cycles of lengths greater than or equal to 2 uniquely determined by $\sigma$, up to reordering, and called the constituents of $\sigma$. The order reversing permutation in $S_{n}$ is the permutation $\rho_{0} \in S_{n}$ defined, for every $r \in \llbracket n \rrbracket$, by $\rho_{0}(r)=n-r+1$. Obviously, we have $\left|\rho_{0}\right|=2$ so that $\Omega=\left\{\right.$ id, $\left.\rho_{0}\right\}$ is a subgroup of $S_{n}$. Note that $\rho_{0}$ has exactly one fixed point when $n$ is odd and no fixed point when $n$ is even. Note also that $\Omega$ is an abelian group which admits as unique subgroups $\{\mathrm{id}\}$ and $\Omega$.

Let $\sigma \in S_{n}$. Given $W \in \mathbb{P}(\llbracket n \rrbracket)$, we denote the image of $W$ through $\sigma$ by $\sigma W$ (instead of $\sigma(W)$ ). Moreover, given $\mathbb{W} \subseteq \mathbb{P}(\llbracket n \rrbracket)$, we define $\sigma \mathbb{W}=\{\sigma W \in \mathbb{P}(\llbracket n \rrbracket)$ : $W \in \mathbb{W}\}$. Note that, for every $\sigma_{1}, \sigma_{2} \in S_{n}, W \in \mathbb{P}(\llbracket n \rrbracket)$ and $\mathbb{W} \subseteq \mathbb{P}(\llbracket n \rrbracket)$, we have that $\left(\sigma_{2} \sigma_{1}\right) W=\sigma_{2}\left(\sigma_{1} W\right)$ and $\left(\sigma_{2} \sigma_{1}\right) \mathbb{W}=\sigma_{2}\left(\sigma_{1} \mathbb{W}\right)$. Thus, brackets can be omitted

$\overline{10}$ Given $A, B$ and $C$ sets and $f: A \rightarrow B$ and $g: B \rightarrow C$ functions, we denote by $g f$ the right-to-left composition of $f$ and $g$, that is, the function from $A$ to $C$ defined, for every $a \in A$, as $g f(a)=g(f(a))$. 
in this type of writings. Given $R \in \mathbf{R}(\llbracket n \rrbracket)$, we set

$$
\sigma R=\left\{(\sigma(x), \sigma(y)) \in \llbracket n \rrbracket^{2}:(x, y) \in R\right\}, \quad R \rho_{0}=\left\{(y, x) \in \llbracket n \rrbracket^{2}:(x, y) \in R\right\},
$$

and $R$ id $=R$. In other words, for every $x, y \in \llbracket n \rrbracket, x \succeq_{R} y$ if and only if $\sigma(x) \succeq_{\sigma R}$ $\sigma(y) ; x \succeq_{R} y$ if and only if $y \succeq_{R \rho_{0}} x$. Given $\mathbf{Q} \subseteq \mathbf{R}(\llbracket n \rrbracket)$ and $\rho \in \Omega$, we also set

$$
\sigma \mathbf{Q}=\{\sigma R \in \mathbf{R}(\llbracket n \rrbracket): R \in \mathbf{Q}\}, \quad \mathbf{Q} \rho=\{R \rho \in \mathbf{R}(\llbracket n \rrbracket): R \in \mathbf{Q}\} .
$$

Note that, for every $\sigma_{1}, \sigma_{2} \in S_{n}, \rho_{1}, \rho_{2} \in \Omega, R \in \mathbf{R}(\llbracket n \rrbracket)$ and $\mathbf{Q} \subseteq \mathbf{R}(\llbracket n \rrbracket)$, we have that $\left(\sigma_{2} \sigma_{1}\right) R=\sigma_{2}\left(\sigma_{1} R\right), R\left(\rho_{1} \rho_{2}\right)=\left(R \rho_{1}\right) \rho_{2},\left(\sigma_{1} R\right) \rho_{1}=\sigma_{1}\left(R \rho_{1}\right)$ and $\left(\sigma_{2} \sigma_{1}\right) \mathbf{Q}=$ $\sigma_{2}\left(\sigma_{1} \mathbf{Q}\right), \mathbf{Q}\left(\rho_{1} \rho_{2}\right)=\left(\mathbf{Q} \rho_{1}\right) \rho_{2},\left(\sigma_{1} \mathbf{Q}\right) \rho_{1}=\sigma_{1}\left(\mathbf{Q} \rho_{1}\right)$. Those properties allow to avoid brackets when writing such kinds of products.

\section{Preference relations and preference profiles}

From now on, let $n \in \mathbb{N}$ with $n \geq 2$ be fixed, and let $N=\llbracket n \rrbracket$ be the set of names of alternatives.

A preference relation on $N$ is a linear order on $N$. Let $q \in \mathbf{L}(N)$ be a preference relation. If $x, y \in N$ are alternatives, we interpret the writing $x \succeq_{q} y$ by saying that $x$ is at least as good as $y$ (according to $q$ ), and the writing $x \succ_{q} y$ by saying that $x$ is preferred to $y$ (according to $q$ ). Note that, since $q$ is a linear order, $x \succ_{q} y$ is equivalent to $x \neq y$ and $x \succeq_{q} y$. It is well known that there exists a unique numbering $x_{1}, x_{2}, \ldots, x_{n}$ of the distinct elements in $N$ such that, once set $R=\left\{\left(x_{i}, x_{i+1}\right) \in\right.$ $\left.N^{2}: i \in\{1, \ldots, n-1\}\right\} \in \mathbf{R}(N)$, we have $q \supseteq R$ and $q$ is the only linear order containing the relation $R$. Thus, we can completely identify $q$ with its subset $R$, which we write in the form $x_{1} \succ_{q} x_{2} \succ_{q} \cdots \succ_{q} x_{n}$. We refer to $r$ as the rank of $x_{r}$ in $q$. Since the map from $\llbracket n \rrbracket$ to $\llbracket n \rrbracket$ which associates with any $r \in \llbracket n \rrbracket$ the alternative $x_{r}$ is a bijection and thus an element of $S_{n}$, we also have that $q$ is completely identified with such permutation, which we continue to call $q$. Explicitly, if $q$ is interpreted in $S_{n}$, then $q(r)=x_{r}$ for all $r \in \llbracket n \rrbracket$. In this way, we have established a well-known and remarkable identification of $\mathbf{L}(N)$ with $S_{n}$. Note now that if $\psi \in S_{n}$, then the relations $\psi q$ and $q \rho_{0}$, as defined in (5), are the linear orders given by $\psi\left(x_{1}\right) \succ_{\psi q}$ $\psi\left(x_{2}\right) \succ_{\psi q} \cdots \succ_{\psi q} \psi\left(x_{n}\right)$ and $x_{n} \succ_{q \rho_{0}} x_{n-1} \succ_{q \rho_{0}} \cdots \succ_{q \rho_{0}} x_{1}$. In particular, for every $\psi \in S_{n}$ and $\rho \in \Omega, \psi q$ and $q \rho$ can be interpreted as products of permutations in the symmetric group $S_{n}$. As a consequence, thanks to the cancellation law in $S_{n}$, we have that, for every $\psi_{1}, \psi_{2} \in S_{n}$ and $\rho_{1}, \rho_{2} \in \Omega, \psi_{1} q=\psi_{2} q$ implies $\psi_{1}=\psi_{2}$ and $q \rho_{1}=q \rho_{2}$ implies $\rho_{1}=\rho_{2}$. Moreover, by elementary properties of the symmetric group, we have that $\psi \mathbf{L}(N) \rho=\mathbf{L}(N)$ for all $\psi \in S_{n}$ and $\rho \in \Omega$.

From now on, let $h \in \mathbb{N}$ with $h \geq 2$ be fixed, and let $H=\llbracket h \rrbracket$ be the set of names of individuals. A preference profile is an element of $\mathbf{L}(N)^{h}$. The set $\mathbf{L}(N)^{h}$ is denoted by $\mathcal{P}$. If $p \in \mathcal{P}$ and $i \in H$, the $i$ th component $p_{i}$ of $p$ represents the preferences of individual $i$. 
Let us set now

$$
G=S_{h} \times S_{n} \times \Omega
$$

Then, $G$ is a group through component-wise multiplication, that is, defining, for every $\left(\varphi_{1}, \psi_{1}, \rho_{1}\right) \in G$ and $\left(\varphi_{2}, \psi_{2}, \rho_{2}\right) \in G,\left(\varphi_{1}, \psi_{1}, \rho_{1}\right)\left(\varphi_{2}, \psi_{2}, \rho_{2}\right)=$ $\left(\varphi_{1} \varphi_{2}, \psi_{1} \psi_{2}, \rho_{1} \rho_{2}\right)$. For every $(\varphi, \psi, \rho) \in G$ and $p \in \mathcal{P}$, define $p^{(\varphi, \psi, \rho)} \in \mathcal{P}$ as the preference profile such that, for every $i \in H$,

$$
\left(p^{(\varphi, \psi, \rho)}\right)_{i}=\psi p_{\varphi^{-1}(i)} \rho .
$$

Thus, the preference profile $p^{(\varphi, \psi, \rho)}$ is obtained by $p$ according to the following rules (to be applied in any order): For every $i \in H$, individual $i$ is renamed $\varphi(i)$; for every $x \in N$, alternative $x$ is renamed $\psi(x)$; for every $r \in \llbracket n \rrbracket$, alternatives whose rank is $r$ are moved to rank $\rho(r)$.

For further details and examples on these issues, the reader is referred to Bubboloni and Gori (2016b, Sects. 2.2 and 2.3).

\section{Social preference and social choice correspondences}

A social preference correspondence (SPC) is a correspondence from $\mathcal{P}$ to $\mathbf{L}(N)$. The set of the sPCs is denoted by $\mathfrak{P}$. Thus, if $C \in \mathfrak{P}$ and $p \in \mathcal{P}$, then $C(p)$ is a subset of $\mathbf{L}(N)$.

From now on, let $k \in \llbracket n-1 \rrbracket$ be fixed. A $k$-multiwinner social choice correspondence $\left(k\right.$-SCC) is a correspondence from $\mathcal{P}$ to $\mathbb{P}_{k}(N)$. The set of the $k$-SCCs is denoted by $\mathfrak{C}_{k}$. Thus, if $C \in \mathfrak{C}_{k}$ and $p \in \mathcal{P}$, then $C(p)$ is a set of $k$-subsets of $N$.

We say that $C \in \mathfrak{P}\left(C \in \mathfrak{C}_{k}\right)$ is decisive if, for every $p \in \mathcal{P}, C(p) \neq \varnothing$; resolute if, for every $p \in \mathcal{P},|C(p)|=1$. We say that $C^{\prime} \in \mathfrak{P}\left(C^{\prime} \in \mathfrak{C}_{k}\right)$ is a refinement of $C \in \mathfrak{P}\left(C \in \mathfrak{C}_{k}\right)$ if, for every $p \in \mathcal{P}, C^{\prime}(p) \subseteq C(p)$. Of course, $C$ admits a resolute refinement if and only if $C$ is decisive; $C$ admits a unique resolute refinement if and only if $C$ is resolute.

Let $U$ be a subgroup of $G$. We say that $C \in \mathfrak{P}$ is $U$-symmetric if, for every $p \in \mathcal{P}$ and $(\varphi, \psi, \rho) \in U$, we have

$$
C\left(p^{(\varphi, \psi, \rho)}\right)=\psi C(p) \rho
$$

We say that $C \in \mathfrak{P}\left(C \in \mathfrak{C}_{k}\right)$ is $U$-consistent if, for every $p \in \mathcal{P}$ and $(\varphi, \psi, \rho) \in U$, we have

$$
C\left(p^{(\varphi, \psi, \rho)}\right)=\psi C(p) \quad \text { if } \rho=\mathrm{id}
$$

and

$$
C\left(p^{(\varphi, \psi, \rho)}\right) \neq \psi C(p) \quad \text { if } \rho=\rho_{0} \quad \text { and } \quad|C(p)|=1 .
$$

We stress that the writings in (7), (8) and (9) are meaningful due to the definitions of products between permutations and sets (sets of sets, relations, sets of relations) and their properties described in Sect. 2. The set of $U$-symmetric SPCs is denoted by $\mathfrak{P}^{*}$; 
the set of $U$-consistent SPCs ( $k$-SCCs) is denoted by $\mathfrak{P}^{U}\left(\mathfrak{C}_{k}^{U}\right)$. Observe that we do not introduce the concept of $U$-symmetry for $k$-SCCs. Note also that, if $U^{\prime} \leq U \leq G$, then $\mathfrak{P}^{* U} \subseteq \mathfrak{P}^{* U^{\prime}}, \mathfrak{P}^{U} \subseteq \mathfrak{P}^{U^{\prime}}$ and $\mathfrak{C}_{k}^{U} \subseteq \mathfrak{C}_{k}^{U^{\prime}}$. Some basic links between the concepts of symmetry and consistency are given by the following proposition whose proof is in "Appendix A."

Proposition 1 Let $U \leq G$. Then, the following facts hold true:

(i) $\mathfrak{P}^{* U} \subseteq \mathfrak{P}^{U}$.

(ii) If $U \leq S_{h} \times S_{n} \times\{\mathrm{id}\}$, then $\mathfrak{P}^{* U}=\mathfrak{P}^{U}$.

The concepts of symmetry and consistency with respect to a subgroup $U$ of $G$ include some classic requirements for SPCs ( $k$-SCCs). Indeed, we have that $C \in \mathfrak{P}$ $\left(C \in \mathfrak{C}_{k}\right)$ is anonymous if and only if it is $S_{h} \times\{\mathrm{id}\} \times\{\mathrm{id}\}$-consistent; $C \in \mathfrak{P}\left(C \in \mathfrak{C}_{k}\right)$ is neutral if and only if it is $\{\mathrm{id}\} \times S_{n} \times\{\mathrm{id}\}$-consistent; $C \in \mathfrak{P}\left(C \in \mathfrak{C}_{k}\right)$ is immune to the reversal bias if and only if it is $\{$ id $\} \times\{$ id $\} \times \Omega$-consistent; $C \in \mathfrak{P}$ is reversal symmetric if and only if it is $\{\mathrm{id}\} \times\{\mathrm{id}\} \times \Omega$-symmetric. Moreover, any combination of the properties above mentioned can be interpreted in terms of $U$-symmetry or $U$ consistency where the subgroup $U$ of $G$ is naturally built as described by the next propositions. Their proof is given in "Appendix A." In what follows, given $U_{1}$ and $U_{2}$ subgroups of $G$, we denote by $\left\langle U_{1}, U_{2}\right\rangle$ the subgroup of $G$ generated by $U_{1}$ and $U_{2} .{ }^{11}$

Proposition 2 Let $U_{1}, U_{2} \leq$. Then $\mathfrak{P}^{* U_{2}} \cap \mathfrak{P}^{* U_{2}}=\mathfrak{P}^{*\left\langle U_{1}, U_{2}\right\rangle}$.

Proposition 3 Let $U_{1}, U_{2} \leq G$ such that, for every $i \in\{1,2\}, U_{i}=Z_{i} \times R_{i}$ for some $Z_{i} \leq S_{h} \times S_{n}$ and $R_{i} \leq \Omega$. Then $\mathfrak{P}^{U_{1}} \cap \mathfrak{P}^{U_{2}}=\mathfrak{P}^{\left\langle U_{1}, U_{2}\right\rangle}$ and $\mathfrak{C}_{k}^{U_{1}} \cap \mathfrak{C}_{k}^{U_{2}}=\mathfrak{C}_{k}^{\left\langle U_{1}, U_{2}\right\rangle}$.

In particular, $C \in \mathfrak{P}$ is anonymous, neutral and reversal symmetric if and only if $C$ is $G$-symmetric; $C \in \mathfrak{P}\left(C \in \mathfrak{C}_{k}\right)$ is anonymous, neutral and immune to the reversal bias if and only if $C$ is $G$-consistent. Classic SPCs provide examples of SPCs which are $G$-symmetric or at least $S_{h} \times S_{n} \times\{\mathrm{id}\}$-symmetric. For instance, the Borda and the Copeland SPCs are $G$-symmetric while the Minimax SPC is generally only $S_{h} \times S_{n} \times\{$ id $\}$-symmetric. ${ }^{12}$ Note also that the SPC associating with every $p \in \mathcal{P}$ the whole set $\mathbf{L}(N)$ is surely $G$-symmetric. Such a SPC is called the trivial SPC and, obviously, every $C \in \mathfrak{P}$ is a refinement of it. In particular, every $C \in \mathfrak{P}$ can be interpreted as a refinement.

We finally observe that there is a natural way to construct a $k$-SCC starting from a SPC. Indeed, given $C \in \mathfrak{P}$, we consider the $k$-SCC $C_{k}$ defined, for every $p \in \mathcal{P}$, by

$$
C_{k}(p)=\left\{\{q(r): 1 \leq r \leq k\} \in \mathbb{P}_{k}(N): q \in C(p)\right\} .
$$

Note that $\{q(r): 1 \leq r \leq k\}$ is the set of alternatives ranked by $q$ in the first $k$ positions. Thus, $C_{k}(p)$ selects the $k$-subsets formed by the best $k$ alternatives in each winning ranking selected by $C(p) . C_{k}$ is called the $k$-SCC induced by $C$. Induced $k$-SCCs are commonly used in decision making when trying to adapt SPCs to the $k$-multiwinner

\footnotetext{
11 See Jacobson (1974, Section 1.5).

12 See Bubboloni and Gori (2016b).
} 
selection setting. For instance, $k$-SCCs induced by the Kemeny and the ranked pairs SPCs are often considered in the literature.

Of course, given $C \in \mathfrak{P}, C$ is decisive if and only if $C_{k}$ is decisive; if $C$ is resolute, then $C_{k}$ is resolute. Moreover, if $C^{\prime} \in \mathfrak{P}$ is a refinement of $C$, then $C_{k}^{\prime}$ is a refinement of $C_{k}$. The following proposition, whose proof is in "Appendix A," expresses the main basic property of the induced $k$-SCC with respect to symmetry.

Proposition 4 Let $U \leq G$. If $C \in \mathfrak{P}^{* U}$, then $C_{k} \in \mathfrak{C}_{k}^{U}$.

In particular, the $k$-SCCs induced by the Borda, the Copeland and the trivial SPCs are $G$-consistent, while the one induced by the Minimax SPC is $S_{h} \times S_{n} \times\{\mathrm{id}\}$-consistent. Note also that the $k$-SCC induced by the trivial SPC associates, with every $p \in \mathcal{P}$, the set of all the possible $k$-subsets of $N$. We refer to that induced $k$-SCC as the trivial $k$-SCC and, obviously, every $C \in \mathfrak{C}_{k}$ is a refinement of it. In particular, every $C \in \mathfrak{C}_{k}$ can be interpreted as a refinement.

\subsection{Social methods}

Let us introduce now a final new concept which will be very important in the sequel. A social method is a function from $\mathcal{P}$ to $\mathbf{R}(N)$. The set of social methods is denoted by $\mathfrak{M}$. Let $R \in \mathfrak{M}$. We say that $R$ is acyclic (transitive, complete, etc.) if, for every $p \in \mathcal{P}$, the relation $R(p)$ is acyclic (transitive, complete, etc.). The SPC associated with $R$, denoted by $C^{R}$, is defined, for every $p \in \mathcal{P}$, by

$$
C^{R}(p)=\{q \in \mathbf{L}(N): R(p) \subseteq q\} .
$$

Note that, by the well-known Szpilrajn's extension theorem (Szpilrajn 1930), $C^{R}$ is decisive if and only if $R$ is acyclic.

Given $U \leq G$, we say that $R$ is $U$-symmetric if, for every $p \in \mathcal{P}$ and $(\varphi, \psi, \rho) \in U$,

$$
R\left(p^{(\varphi, \psi, \rho)}\right)=\psi R(p) \rho .
$$

We stress that the above writing is meaningful due to the definitions of products between permutations and relations described in Sect. 2. The set of $U$-symmetric social methods is denoted by $\mathfrak{M}^{* U}$.

Note that if $U^{\prime} \leq U$ and $R$ is $U$-symmetric, then $R$ is also $U^{\prime}$-symmetric. Moreover, as proved in "Appendix A," the next propositions hold true.

Proposition 5 Let $U \leq G$. If $R \in \mathfrak{M}^{* U}$, then $C^{R} \in \mathfrak{P}^{* U}$.

Proposition 6 Let $U_{1}, U_{2} \leq G$. Then $\mathfrak{M}^{* U_{1}} \cap \mathfrak{M}^{* U_{2}}=\mathfrak{M}^{*\left\langle U_{1}, U_{2}\right\rangle}$.

As an example, consider the social method $R$ defined, for every $p \in \mathcal{P}$, by

$$
R(p)=\left\{(x, y) \in N^{2}: \forall i \in H, x \succ_{p_{i}} y\right\},
$$

and note that $R \in \mathfrak{M}^{* G}$. The SPC associated with it is called the Pareto SPC and, as wellknown, it is decisive. The $k$-SCC induced by the Pareto SPC is called the Pareto $k$-SCC. 
Note that, by Proposition 5, the Pareto SPC is $G$-symmetric and thus, by Proposition 4, the Pareto $k$-SCC is $G$-consistent.

\section{Main results}

Given a SPC or a $k$-SCC, our main purpose is to find a resolute refinement of it satisfying suitable symmetry or consistency properties. More precisely, we try to find an answer to the next three questions:

- Given a SPC $C$ and $U \leq G$, can we find a resolute refinement of $C$ which is $U$-symmetric?

- Given a SPC $C$ and $U \leq G$, can we find a resolute refinement of $C$ which is $U$-consistent?

- Given a $k$-SCC $C$ and $U \leq G$, can we find a resolute refinement of $C$ which is $U$-consistent?

Indeed, for each of the above questions, we provide conditions on $C$ and $U$ which allow to give an affirmative answer. We emphasize that, assuming $C$ to be trivial, the three questions above are about the existence of resolute $U$-symmetric SPCs, $U$-consistent SPCs and $U$-consistent $k$-SCCs.

In order to describe our main results, we need to recall the crucial concept of regular subgroup of $G$, introduced in Bubboloni and Gori (2015). A subgroup $U$ of $G$ is said to be regular if, for every $p \in \mathcal{P}$, there exists $\psi_{*} \in S_{n}$ conjugate to $\rho_{0}$ such that

$$
\operatorname{Stab}_{U}(p) \subseteq\left(S_{h} \times\{\operatorname{id}\} \times\{\operatorname{id}\}\right) \cup\left(S_{h} \times\left\{\psi_{*}\right\} \times\left\{\rho_{0}\right\}\right)
$$

where $\operatorname{Stab}_{U}(p)=\left\{(\varphi, \psi, \rho) \in U: p^{(\varphi, \psi, \rho)}=p\right\}$

We also recall the fundamental result about those groups proved in Bubboloni and Gori (2015, Theorem 7):

There exists a $U$-symmetric resolute SPC if and only if $U$ is regular.

Thus, if we want to focus on $U$-symmetric resolute refinements of a given SPC, we necessarily have to assume $U$ regular. The next result is a generalization of Theorem 11 in Bubboloni and Gori (2015). Its proof is proposed in "Appendix B."

Theorem 7 Let $U$ be a regular subgroup of $G$ and $C$ be a decisive and $U$-symmetric SPC. Then, the three following conditions are equivalent:

(i) $C$ admits a $U$-symmetric resolute refinement;

(ii) there exists an irreflexive and acyclic $U$-symmetric social method $R$ such that $C^{R}$ refines $C$;

(iii) there exists an irreflexive and acyclic social method $R$ such that $C^{R}$ refines $C$ and the following condition is satisfied:

(a) for every $p \in \mathcal{P}, x, y \in N$ and $\left(\varphi, \psi, \rho_{0}\right) \in \operatorname{Stab}_{U}(p)$, we have that $(x, y) \in$ $R(p)$ if and only if $(\psi(y), \psi(x)) \in R(p)$. 
Note that, for every $U \leq G$, there exist a lot of decisive and $U$-symmetric sPCs. For instance, the trivial, the Pareto, the Borda and the Kemeny SPCs are decisive and $G$-symmetric, so that they are $U$-symmetric too. Theorem 7 may appear cryptic and awkward, due to its very formal appearance. Fortunately, it immediately reveals its concrete applicative power.

Corollary 8 Let $U$ be a regular subgroup of $G$ with $U \leq S_{h} \times S_{n} \times\{\mathrm{id}\}$ and $C$ be a decisive and $U$-symmetric SPC. Then, $C$ admits a $U$-symmetric resolute refinement.

Proof For every $p \in \mathcal{P}$, we have $C(p) \neq \varnothing$. Choose then, for every $p \in \mathcal{P}, q_{p} \in$ $C(p)$. Since $q_{p} \in \mathbf{L}(N) \subseteq \mathbf{R}(N)$, we define $R: \mathcal{P} \rightarrow \mathbf{R}(N)$ setting, for every $p \in \mathcal{P}, R(p)=q_{p} \backslash \Delta$, where $\Delta=\{(x, x): x \in N\}$. Then $R$ is an irreflexive and acyclic social method. Moreover, $C^{R}(p)=\left\{q \in \mathbf{L}(N): q_{p} \backslash \Delta \subseteq q\right\}=\left\{q_{p}\right\}$, so that $C^{R}$ refines $C$. Since (a) trivially holds, we have that condition (iii) in Theorem 7 is satisfied. Therefore, by Theorem 7, $C$ admits a $U$-symmetric resolute refinement.

The concept of regular group works properly even to analyze consistency. Theorems 9 below is proved in "Appendix B" by widely extending the theory developed in Bubboloni and Gori (2016b) to the framework here considered.

Theorem 9 Let $U$ be a regular subgroup of $G$ and $C$ be a decisive and $U$-consistent SPC. Then, $C$ admits a $U$-consistent resolute refinement.

Theorem 10 below, also proved in "Appendix B," is a largely unexpected result. It establishes a deep link between the number $n$ of alternatives and the number $k$ of winners to be selected in order to make each decisive and $U$-consistent $k$-SCC admit a $U$-consistent resolute refinement. It points out that there is a substantial difference between SPCs and $k$-SCCs with respect to the existence of $U$-consistent resolute refinements. Indeed, while for SPCs such existence is guaranteed for every $U$-consistent SPC once $U$ is regular (Theorem 9), for $k$-SCCs one needs the fulfillment of a further restrictive condition in addition to regularity.

Theorem 10 Let $U$ be a regular subgroup of $G$. Then, the two following facts are equivalent:

(i) every decisive and $U$-consistent $k$-SCC admits a $U$-consistent resolute refinement;

(ii) one of the following conditions is satisfied:

(a) for every $\left(\varphi, \psi, \rho_{0}\right) \in U, \psi$ is not a conjugate of $\rho_{0}$,

(b) $n \leq 3$,

(c) $k \in\{1, n-1\}$,

(d) $n$ is even and $k$ is odd.

Since condition (a) in Theorem 10 certainly holds when $U \leq S_{h} \times S_{n} \times\{$ id $\}$, we immediately obtain the following useful consequence.

Corollary 11 Let $U$ be a regular subgroup of $G$, with $U \leq S_{h} \times S_{n} \times\{\mathrm{id}\}$, and $C$ be a decisive and $U$-consistent $k$-SCC. Then $C$ admits a $U$-consistent resolute refinement. 
Observe that condition (a) in Theorem 10 can be satisfied also by some regular subgroups not included in $S_{h} \times S_{n} \times\{$ id $\}$. Consider the group $\{$ id $\} \times\{$ id $\} \times \Omega$ and note that, for every $p \in \mathcal{P}$, $\operatorname{Stab}_{U}(p)=\{(\mathrm{id}, \mathrm{id}, \mathrm{id})\} .{ }^{13}$ Thus, $\{\mathrm{id}\} \times\{\mathrm{id}\} \times \Omega$ is regular and, since id is not conjugate to $\rho_{0}$, (a) trivially holds. We end the section by stating two results about the induced $k$-SCCs.

Proposition 12 Let $U$ be a regular subgroup of $G$, with $U \leq S_{h} \times S_{n} \times\{\mathrm{id}\}$, and $C$ be a decisive and $U$-consistent SPC. Then the induced $k$-SCC $C_{k}$ admits a $U$-consistent resolute refinement.

Proof Since $U \leq S_{h} \times S_{n} \times\{\mathrm{id}\}$, we have that $C$ is $U$-symmetric and thus, by Proposition $4, C_{k}$ is a decisive $U$-consistent $k$-SCC. Thus, by Corollary $11, C_{k}$ admits a $U$-consistent resolute refinement.

Proposition 13 Let $U \leq G$ and $C$ be a decisive SPC. If $C$ admits a resolute $U$-symmetric refinement, then $U$ is regular and the induced $k$-SCC $C_{k}$ admits a $U$ consistent resolute refinement.

Proof Let $f$ be a resolute $U$-symmetric refinement of $C$. By (11), $U$ is regular. Moreover, by Proposition $4, f_{k}$ is $U$-consistent. On the other hand, clearly, $f_{k}$ is resolute and refines $C_{k}$.

\section{Regular groups}

Because of the results presented in Sect. 5, the importance of the regular subgroups of $G$ is evident. In this section, we propose some theorems which provide a way to test whether a subgroup $U$ of $G$ of the type $V \times W \times\{$ id $\}$ or $V \times W \times \Omega$, with $V \leq S_{h}$ and $W \leq S_{n}$, is regular or not. These types of groups are particularly relevant for applications. Regular groups of a different structure and still of interest in the applications will be treated in Sect. 8.3.

We start with some preliminary definitions. Let $m \in \mathbb{N}$ be fixed in this section. Consider $\sigma \in S_{m}$. For every $x \in \llbracket m \rrbracket$, the $\sigma$-orbit $x^{\langle\sigma\rangle}$ of $x$ is defined by $x^{\langle\sigma\rangle}=$ $\left\{\sigma^{t}(x) \in \llbracket m \rrbracket: t \in \mathbb{N}\right\}$. It is well known that $\left|x^{\langle\sigma\rangle}\right|=s$ where $s=\min \{t \in \mathbb{N}$ : $\left.\sigma^{t}(x)=x\right\}$. If $x$ is fixed by $\sigma$, then $x^{\langle\sigma\rangle}=\{x\}$. If instead $x$ is moved by $\sigma$ and the constituent of $\sigma$ moving $x$ is the cycle $\left(x_{1} \cdots x_{s}\right)$, then $x^{\langle\sigma\rangle}=\left\{x_{1}, \ldots, x_{s}\right\}$. The set $O(\sigma)=\left\{x^{\langle\sigma\rangle}: x \in \llbracket m \rrbracket\right\}$ of the $\sigma$-orbits is a partition of $\llbracket m \rrbracket$, and we denote its size by $r(\sigma)$. A system of representatives of the $\sigma$-orbits is a set $\left\{x_{1}, \ldots, x_{r(\sigma)}\right\} \in \mathbb{P}_{r(\sigma)}(\llbracket m \rrbracket)$ such that $O(\sigma)=\left\{x_{1}^{\langle\sigma\rangle}, \ldots, x_{r(\sigma)}^{\langle\sigma\rangle}\right\}$.

Next we recall the well-known number theoretical concept of partition. A partition of $m$ is an unordered list $T=\left[m_{1}, \ldots, m_{r}\right]$ where $r \in \mathbb{N}$, for every $j \in\{1, \ldots, r\}$, $m_{j} \in \mathbb{N}$ and $m=\sum_{j=1}^{r} m_{j}$. The numbers $m_{1}, \ldots, m_{r}$ are called the terms of $T$. The set of partitions of $m$ is denoted by $\Pi_{m}$.

Consider $T \in \Pi_{m}$ and assume that $T$ admits $s \in \mathbb{N}$ distinct terms, say $m_{1}<$ $\cdots<m_{s}$, and assume that, for every $j \in \llbracket s \rrbracket, m_{j}$ appears $t_{j} \geq 1$ times in $T$. Then,

13 Indeed $p^{\left(\mathrm{id}, \mathrm{id}, \rho_{0}\right)}=p$ implies $p_{1} \rho_{0}=p_{1}$, which by cancellation law in the symmetric group, gives the contradiction $\rho_{0}=\mathrm{id}$. 
we use the notation $T=\left[m_{1}^{t_{1}}, \ldots, m_{s}^{t_{s}}\right]$ (where $t_{j}$ is omitted when it equals 1 ). We say that $\left[m_{1}^{t_{1}}, \ldots, m_{s}^{t_{s}}\right]$ is the normal form of $T$ and that $t_{j}$ is the multiplicity of $m_{j}$. For instance, $[2,1,3,1] \in \Pi_{7}$ has normal form $\left[1^{2}, 2,3\right]$. We recall the well-known surjective function

$$
T: S_{m} \rightarrow \Pi_{m}, \quad \sigma \mapsto T(\sigma)=\left[\left|x_{1}^{\langle\sigma\rangle}\right|, \ldots,\left|x_{r(\sigma)}^{\langle\sigma\rangle}\right|\right]
$$

where $\left\{x_{1}, \ldots, x_{r(\sigma)}\right\} \in \mathbb{P}_{r(\sigma)}(\llbracket m \rrbracket)$ is a system of representatives of the $\sigma$-orbits. For every $\sigma \in S_{m}, T(\sigma)$ is called the type of $\sigma$. In other words, $T(\sigma)$ is the unordered list of the sizes of the orbits of the group generated by $\sigma$ on the set $\llbracket m \rrbracket$. Note that the number of terms equal to 1 in $T(\sigma)$ counts the fixed points of $\sigma$ while the number of terms different from 1 counts the constituents of $\sigma$. Moreover, $|\sigma|=\operatorname{lcm}(T(\sigma))$. For instance, if $\sigma=(123)(456)(78) \in S_{9}$, then $r(\sigma)=4$, the type of $\sigma$ is $T(\sigma)=$ $[1,2,3,3] \in \Pi_{9},|\sigma|=\operatorname{lcm}[1,2,3,3]=6$ and a system of representatives of the $\sigma$-orbits is $\{1,4,7,9\} \in \mathbb{P}_{4}(\llbracket 9 \rrbracket)$. The theoretical importance of the concept of type relies on the fact that two permutations are conjugate if and only if they have the same type.

Given $U \leq S_{m}$, we define the type number of $U$ by

$$
\gamma(U)=\operatorname{lcm}\{\operatorname{gcd}(T(\sigma)): \sigma \in U\}
$$

We describe some basic properties of the type number after having introduced some arithmetic notation. Let $x, y \in \mathbb{N}$. If $x$ divides $y$, we write $x \mid y$. If $\pi \in \mathbb{N}$ is a prime number we denote by $x_{\pi}=\max \left\{\pi^{a}: a \in \mathbb{N}_{0}, \pi^{a} \mid x\right\}$ the $\pi$-part of $x$.

Lemma 14 Let $U, V \leq S_{m}$. Then, the following facts hold true.

(i) If $U \leq V$, then $\gamma(U) \mid \gamma(V)$.

(ii) $\gamma(U) \mid m$.

(iii) If $U$ contains an $m$-cycle, then $\gamma(U)=m$. In particular, $\gamma\left(S_{m}\right)=m$.

Proof (i) The set of integers $\{\operatorname{gcd}(T(\sigma)): \sigma \in U\}$ is included in the set of integers $\{\operatorname{gcd}(T(\sigma)): \sigma \in V\}$, and thus, the least common multiple of the first divides that of the second.

(ii) Let $\sigma \in U$ and let $T(\sigma)=\left[m_{1}, \ldots, m_{r}\right]$. If $d=\operatorname{gcd}(T(\sigma))$, we have that $d \mid m_{j}$ for all $j \in \llbracket r \rrbracket$ and since $\sum_{j=1}^{r} m_{j}=m$ we have $d \mid m$. Thus, $m$ is a common multiple for the integers in $\{\operatorname{gcd}(T(\sigma)): \sigma \in U\}$, which implies $\gamma(U) \mid m$.

(iii) Let $\sigma \in U$ be an $m$-cycle. Then, $T(\sigma)=[m]$ and $\operatorname{gcd}(T(\sigma))=m$. Thus, $m \mid \gamma(U)$. Since by (ii) we also have $\gamma(U) \mid m$, we conclude that $\gamma(U)=m$.

Theorem 15 Let $V \leq S_{h}$ and $W \leq S_{n}$. Then, $V \times W \times\{\mathrm{id}\}$ is regular if and only if

$$
\operatorname{gcd}(\gamma(V),|W|)=1
$$

Proof Let $U=V \times W \times\{\operatorname{id}\}$. Assume first that $\operatorname{gcd}(\gamma(V),|W|)=1$. Assume further, by contradiction, that $U$ is not regular. Then, by Theorem 12 in Bubboloni and Gori (2015), there exist $(\varphi, \psi, \mathrm{id}) \in U$ and a prime $\pi$ such that $|\psi|_{\pi}>1$ and 
$|\psi|_{\pi} \mid \operatorname{gcd}(T(\varphi))$. Then $\pi \mid \gamma(V)$ and, by Lagrange Theorem, $\pi|| W \mid$ so that $\pi \mid \operatorname{gcd}(\gamma(V),|W|)=1$, a contradiction.

Assume next that $U$ is regular. We show that if $\pi$ is a prime dividing $|W|$, then $\pi \nmid$ $\gamma(V)$. Let $\pi|| W \mid$. Then, by Cauchy Theorem, there exists $\psi \in W$ with $|\psi|=\pi$. But, for every $\varphi \in V$, we have $(\varphi, \psi$, id $) \in U$ and, of course, $|\psi|_{\pi}=\pi$. Thus, by Theorem 12 in Bubboloni and Gori (2015), we have that, for every $\varphi \in V, \pi \nmid \operatorname{gcd}(T(\varphi))$ so that $\pi \nmid \operatorname{lcm}\{\operatorname{gcd}(T(\varphi)): \varphi \in V\}=\gamma(V)$.

Theorem 16 Let $V \leq S_{h}$ and $W \leq S_{n}$. Then $V \times W \times \Omega$ is regular if and only if

$$
\operatorname{gcd}(\gamma(V), \operatorname{lcm}(|W|, 2))=1
$$

Proof Let $U=V \times W \times \Omega$. Assume first that $\operatorname{gcd}(\gamma(V), \operatorname{lcm}(|W|, 2))=1$. Assume further, by contradiction, that $U$ is not regular. Then, by Theorem 12 in Bubboloni and Gori (2015), there exist $(\varphi, \psi$, id $) \in U$ and a prime $\pi$ such that $|\psi|_{\pi}>1$ and $|\psi|_{\pi} \mid \operatorname{gcd}(T(\varphi))$, or there exist $\left(\varphi, \psi, \rho_{0}\right) \in U$, with $\psi^{2}=$ id and $\psi$ not conjugate of $\rho_{0}$, such that $2 \mid \operatorname{gcd}(T(\varphi))$. In the first case, by Lagrange Theorem, we have $\pi|| W \mid$ as well as $\pi \mid \gamma(V)$ and thus $\pi \mid \operatorname{gcd}(\gamma(V), \operatorname{lcm}(|W|, 2))=1$, a contradiction. In the second case, we have $2 \mid \gamma(V)$, which implies the contradiction $2 \mid \operatorname{gcd}(\gamma(V), \operatorname{lcm}(|W|, 2))=1$.

Assume next that $U$ is regular. We show first that if $\pi$ is a prime dividing $|W|$, then $\pi \nmid \gamma(V)$. Let $\pi|| W \mid$. Then, by Cauchy Theorem, there exists $\psi \in W$ with $|\psi|=\pi$. But, for every $\varphi \in V$, we have $(\varphi, \psi$, id $) \in U$ and, of course, $|\psi|_{\pi}=\pi$. Thus, by Theorem 12 in Bubboloni and Gori (2015), we get $\pi \nmid \operatorname{gcd}(T(\varphi))$ and so also $\pi \nmid \operatorname{lcm}\{\operatorname{gcd}(T(\varphi)): \varphi \in V\}=\gamma(V)$. We are then left with proving that $2 \nmid \gamma(V)$, that is, that $2 \nmid \operatorname{gcd}(T(\varphi))$ for all $\varphi \in V$. Let $\varphi \in V$ and consider $\left(\varphi\right.$, id, $\left.\rho_{0}\right) \in U$. Since $\mathrm{id}^{2}=$ id but id is not a conjugate of $\rho_{0}$, by Theorem 12 in Bubboloni and Gori (2015), we get $2 \nmid \operatorname{gcd}(T(\varphi))$.

Among other things, the above theorems show that regularity, even though in principle a very demanding requirement, is quite largely satisfied within the family of subgroups of $G$. Indeed, there are many examples of $V \leq S_{h}$ such that $\gamma(V)=1$ and thus such that $V \times W \times\{\mathrm{id}\}$ and $V \times W \times \Omega$ are regular for any choice of $W \leq S_{n}$. For instance, taking any $V$ isomorphic to $S_{h_{1}} \times S_{h_{2}}$ with $h_{1}, h_{2} \in \mathbb{N}$ such that $h_{1}+h_{2}=h$ and $\operatorname{gcd}\left(h_{1}, h_{2}\right)=1$, one surely get $\gamma(V)=1$.

In order to let the reader understand how concrete could be the description of the regular groups of the type $V \times W \times\{\mathrm{id}\}$ and $V \times W \times \Omega$ given in Theorems 15 and 16, we find now them all in the case $n=h=3$. First note that the complete list of the subgroups of $S_{3}$ is given by

$$
\begin{aligned}
G_{1} & =\{\text { id }\} \\
G_{2} & =\{\text { id },(12)\} \\
G_{3} & =\{\text { id },(13)\} \\
G_{4} & =\{\text { id, }(23)\} \\
G_{5} & =\{\text { id },(123),(132)\} \\
G_{6} & =S_{3} .
\end{aligned}
$$


Table 1 Main regular subgroups in $S_{3} \times S_{3} \times \Omega$

\begin{tabular}{lllll}
\hline $\mathrm{V}$ & $\gamma(V)$ & $|V|$ & $W \leq S_{3}$ with $V \times W \times\{\mathrm{id}\}$ regular & $W \leq S_{3}$ with $V \times W \times \Omega$ regular \\
\hline$G_{1}$ & 1 & 1 & $G_{1}, G_{2}, G_{3}, G_{4}, G_{5}, G_{6}$ & $G_{1}, G_{2}, G_{3}, G_{4}, G_{5}, G_{6}$ \\
$V \in\left\{G_{2}, G_{3}, G_{4}\right\}$ & 1 & 2 & $G_{1}, G_{2}, G_{3}, G_{4}, G_{5}, G_{6}$ & $G_{1}, G_{2}, G_{3}, G_{4}, G_{5}, G_{6}$ \\
$G_{5}$ & 3 & 3 & $G_{1}, G_{2}, G_{3}, G_{4}$ & $G_{1}, G_{2}, G_{3}, G_{4}$ \\
$G_{6}$ & 3 & 6 & $G_{1}, G_{2}, G_{3}, G_{4}$ & $G_{1}, G_{2}, G_{3}, G_{4}$ \\
\hline
\end{tabular}

Thus, in $S_{3} \times S_{3} \times \Omega$ we have 36 different subgroups of the form $V \times W \times\{\mathrm{id}\}$ and 36 different subgroups of the form $V \times W \times \Omega$. The values of $\gamma\left(G_{i}\right)$ and $\left|G_{i}\right|$ are immediately computed for all $i \in\{1, \ldots, 6\}$, and thus, by Theorem 15, we get 32 regular subgroups of type $V \times W \times\{\mathrm{id}\}$ and, by Theorem 16, 32 regular subgroups of type $V \times W \times \Omega$ as shown in Table 1 .

\section{Generalized anonymity and neutrality}

Consider $V \leq S_{h}$ and $W \leq S_{n}$. Given $C \in \mathfrak{P}\left(C \in \mathfrak{C}_{k}\right)$, we say that $C$ is $V$-anonymous if $C$ is $V \times\{$ id $\} \times\{$ id $\}$-consistent; $W$-neutral if $C$ is $\{\mathrm{id}\} \times W \times\{\mathrm{id}\}$-consistent. Thus, $C$ is $V$-anonymous if permuting individual names according to permutations in $V$ has no effect on the final outcome; $C$ is $W$-neutral if the unique effect of permuting alternative names according to a permutation in $W$ is that alternative names are accordingly permuted in the final outcome. Note that, the concepts of $S_{h}$-anonymity and $S_{n}$-neutrality correspond to the classical concepts of anonymity and neutrality, respectively. Note also that every $C \in \mathfrak{P}\left(C \in \mathfrak{C}_{k}\right)$ is $\{\mathrm{id}\}$-anonymous and $\{\mathrm{id}\}$-neutral.

We also stress that, by Propositions 2 and $3, C \in \mathfrak{P}\left(C \in \mathfrak{C}_{k}\right)$ is $V$-anonymous and $W$-neutral if and only if $C$ is $V \times W \times\{$ id $\}$-consistent; $C \in \mathfrak{P}$ is $V$-anonymous, $W$-neutral and reversal symmetric if and only if $C$ is $V \times W \times \Omega$-symmetric; $C \in \mathfrak{P}$ $\left(C \in \mathfrak{C}_{k}\right)$ is $V$-anonymous, $W$-neutral and immune to the reversal bias if and only if $C$ is $V \times W \times \Omega$-consistent.

Below we provide some simple but very important consequences of the theory developed in the previous sections.

Theorem 17 Let $V \leq S_{h}, W \leq S_{n}$ and $C$ be a decisive, $V$-anonymous and $W$-neutral SPC. Then, the two following conditions are equivalent:

(i) $C$ admits a $V$-anonymous and $W$-neutral resolute refinement;

(ii) $\operatorname{gcd}(\gamma(V),|W|)=1$.

Proof (i) $\Rightarrow$ (ii). Assume that (i) holds true. Then, by (11), we have that the group $V \times W \times\{\mathrm{id}\}$ is regular, so that, by Theorem $15, \operatorname{gcd}(\gamma(V),|W|)=1$.

(ii) $\Rightarrow$ (i). Assume that (ii) holds true. Then, by Theorem 15, the group $V \times W \times\{$ id $\}$ is regular. Then we can apply Theorem 9.

Note that condition $\operatorname{gcd}(\gamma(V),|W|)=1$ above is trivially satisfied if one between $V$ and $W$ is trivial. Observe also that the above theorem generalizes the classic result 
about the existence of a resolute anonymous and neutral SPC. Namely, taking $V=S_{h}$, $W=S_{n}$ and using Lemma 14 (iii), we immediately have that there exists a resolute, anonymous and neutral SPC if and only if $\operatorname{gcd}(h, n !)=1$.

Theorem 18 Let $V \leq S_{h}, W \leq S_{n}$ and $C$ be a decisive, $V$-anonymous, $W$-neutral and reversal symmetric SPC. Then, the two following conditions are equivalent:

(i) $C$ admits a $V$-anonymous, $W$-neutral and reversal symmetric resolute refinement;

(ii) $\operatorname{gcd}(\gamma(V), \operatorname{lcm}(|W|, 2))=1$ and there exists an irreflexive acyclic social method $R$ such that $C^{R}$ refines $C$ and, for $U=V \times W \times \Omega$, the following condition is satisfied:

(a) for every $p \in \mathcal{P}, x, y \in N$ and $\left(\varphi, \psi, \rho_{0}\right) \in \operatorname{Stab}_{U}(p)$, we have that $(x, y) \in$ $R(p)$ if and only if $(\psi(y), \psi(x)) \in R(p)$.

Proof (i) $\Rightarrow$ (ii). Assume that (i) holds true. Then, by (11), we have that the group $U$ is regular, so that, by Theorem $16, \operatorname{gcd}(\gamma(V), \operatorname{lcm}(|W|, 2))=1$. Moreover, using now Theorem 7 we conclude the proof.

(ii) $\Rightarrow$ (i). Assume that (ii) holds true. Then, by Theorem 16, we know that the group $U$ is regular. Therefore we can apply Theorem 7 .

Theorem 19 Let $V \leq S_{h}, W \leq S_{n}$ and $C$ be a decisive, $V$-anonymous, $W$-neutral and immune to the reversal bias SPC. If $\operatorname{gcd}(\gamma(V), \operatorname{lcm}(|W|, 2))=1$, then $C$ admits a $V$-anonymous, $W$-neutral and immune to the reversal bias resolute refinement.

Proof By Theorem 16, the group $U=V \times W \times \Omega$ is regular. Then Theorem 9 applies.

Theorem 20 Let $V \leq S_{h}, W \leq S_{n}$ and $C$ be a decisive, $V$-anonymous and $W$-neutral $k$-SCC. If $\operatorname{gcd}(\gamma(V),|W|)=1$, then $C$ admits a $V$-anonymous and $W$-neutral resolute refinement.

Proof Apply Theorem 15 and Corollary 11.

Theorem 21 Let $V \leq S_{h}, W \leq S_{n}$ and assume that $\operatorname{gcd}(\gamma(V), \operatorname{lcm}(|W|, 2))=1$. Then, the two following facts are equivalent:

(i) every decisive, $V$-anonymous, $W$-neutral and immune to the reversal bias $k$ SCC admits a $V$-anonymous, $W$-neutral and immune to the reversal bias resolute refinement;

(ii) one of the following conditions is satisfied:

(a) for every $\psi \in W, \psi$ is not a conjugate of $\rho_{0}$,

(b) $n \leq 3$,

(c) $k \in\{1, n-1\}$,

(d) $n$ is even and $k$ is odd.

Proof Apply Theorem 16 and Theorem 10. 


\section{Some applications}

Consider a committee of $h$ members facing the problem of selecting a set of $k$ alternatives within a set of $n$ alternatives. The committee members need a suitable procedure for determining the $k$ alternatives from their preferences. If they agree to express their preferences via a linear order on the set of alternatives, they are in fact looking for a resolute $k$-SCC. Suppose now that there is a general agreement on the fact that the resolute $k$-SCC to be used should obey to certain fundamental principles which can be summarized in the requirement of being a refinement of a given decisive, anonymous, neutral and immune to the reversal bias $k$-SCC $C .{ }^{14}$

In what follows, we are going to discuss four particular qualifications of the abovedescribed situation where the theory we developed can be applied and turn out to be useful to the committee. We stress that the proposed applications only focus on $k$-SCCs. Suitable adaptations to the framework of SPCs are natural and left to the reader.

\subsection{Grouping individuals and alternatives}

Assume that the particular structure of the committee naturally allows to group committee members into subcommittees and that the typology of the alternatives allows to group them into subclasses. Suppose that committee members also agree on the fact that the desired resolute refinement of $C$ should not distinguish among individuals in the same subcommittee and should equally treat alternatives in the same subclass.

Let $r, s \geq 1$ and assume that $H_{1}, \ldots, H_{r} \subseteq H$ is the list of subcommittees and that $N_{1}, \ldots, N_{s} \subseteq N$ is the list of subclasses so that $\left\{H_{1}, \ldots, H_{r}\right\}$ is a partition of $H$ and $\left\{N_{1}, \ldots, N_{s}\right\}$ is a partition of $N$. Then, the committee needs to find a resolute refinement of $C$ which is $V$-anonymous and $W$-neutral, where

$$
\begin{aligned}
V & =\left\{\varphi \in S_{h}: \forall j \in \llbracket r \rrbracket, \varphi\left(H_{j}\right)=H_{j}\right\}, \\
W & =\left\{\psi \in S_{n}: \forall j \in \llbracket s \rrbracket, \psi\left(N_{j}\right)=N_{j}\right\} .
\end{aligned}
$$

Let us prove now that

$$
\gamma(V)=\operatorname{gcd}\left(\left|H_{1}\right|, \ldots,\left|H_{r}\right|\right)
$$

Indeed, consider $\sigma \in V$ and its type $T(\sigma)$. Let $x \in H_{j}$, for some $j \in \llbracket r \rrbracket$. Since $\sigma\left(H_{j}\right)=H_{j}$, we have that $x^{\langle\sigma\rangle} \subseteq H_{j}$. Thus, the sets $H_{j}$ are union of $\sigma$-orbits. Hence, the partition $T(\sigma)$ of $h$ induces a partition of $\left|H_{j}\right|$ for all $j \in \llbracket r \rrbracket$. It follows that, for every $j \in \llbracket r \rrbracket, \operatorname{gcd}(T(\sigma))$ divides $\left|H_{j}\right|$, and thus, $\operatorname{gcd}(T(\sigma))$ divides $\operatorname{gcd}\left(\left|H_{1}\right|, \ldots,\left|H_{r}\right|\right)$. Then, we also have that $\gamma(V)=\operatorname{lcm}\{\operatorname{gcd}(T(\sigma)): \sigma \in V\}$ divides $\operatorname{gcd}\left(\left|H_{1}\right|, \ldots,\left|H_{r}\right|\right)$. If $\operatorname{gcd}\left(\left|H_{1}\right|, \ldots,\left|H_{r}\right|\right)=1$, then we also have $\gamma(V)=$ $1=\operatorname{gcd}\left(\left|H_{1}\right|, \ldots,\left|H_{r}\right|\right)$. Assume then that $\operatorname{gcd}\left(\left|H_{1}\right|, \ldots,\left|H_{r}\right|\right) \neq 1$. Then, in particular, $\left|H_{j}\right| \neq 1$ for all $j \in \llbracket r \rrbracket$ and it is meaningful to define a cycle $\sigma_{j}$ on its elements. Consider now $\sigma \in S_{h}$ defined by $\sigma=\sigma_{1} \cdots \sigma_{r}$. Then, $T(\sigma)=\left[\left|H_{1}\right|, \ldots,\left|H_{r}\right|\right]$ and $\operatorname{gcd}(T(\sigma))=\operatorname{gcd}\left(\left|H_{1}\right|, \ldots,\left|H_{r}\right|\right)$ divides $\gamma(V)$. Thus, we obtain (14).

\footnotetext{
14 Thus, $C$ might be, for instance, the trivial, the Pareto, the Borda or the Kemeny $k$-SCC. Recall that, those $k$-SCC are $U$-consistent for all $U \leq G$.
} 
Clearly, we also have

$$
|W|=\prod_{j=1}^{s}\left|N_{j}\right| !
$$

Thus, by (14), (15) and Theorem 20, we deduce that if

$$
\operatorname{gcd}\left(\left|H_{1}\right|, \ldots,\left|H_{r}\right|, \prod_{j=1}^{s}\left|N_{j}\right| !\right)=1,
$$

then $C$ admits a $V$-anonymous and $W$-neutral resolute refinement. Moreover, by (14), (15) and Theorem 21, we deduce that if (16) holds true, not all the subclasses are singletons and one of the following conditions is satisfied:

(a) there exist at least two subclasses of odd size,

(b) $n \leq 3$,

(c) $k \in\{1, n-1\}$,

(d) $n$ is even and $k$ is odd,

then $C$ admits a $V$-anonymous, $W$-neutral and immune to the reversal bias resolute refinement.

\subsection{A variation on groupings of individuals and alternatives}

Assume that the particular structure of the committee naturally allows to group committee members into subcommittees and that the typology of the alternatives allows to group them into subclasses as in Sect. 8.1. Suppose, this time, that committee members also agree on the fact that the desired resolute refinement of $C$ should not distinguish among individuals in the same subcommittee, should not distinguish among subcommittees having the same size and should equally treat alternatives in the same subclass.

Using the same notation used in Sect. 8.1, let $H_{1}, \ldots, H_{r}$ be the list of subcommittees and $N_{1}, \ldots, N_{S} \subseteq N$ be the list of subclasses. Then, the committee needs to find a resolute refinement of $C$ which is $V$-anonymous and $W$-neutral, where

$$
V=\left\{\varphi \in S_{h}: \forall j \in \llbracket r \rrbracket, \exists k \in \llbracket r \rrbracket \text { such that } \varphi\left(H_{j}\right)=H_{k}\right\}
$$

and $W$ is as in (13). Defining now, for every $j \in \llbracket r \rrbracket$,

$$
t_{j}=\left|\left\{k \in \llbracket r \rrbracket:\left|H_{k}\right|=\left|H_{j}\right|\right\}\right|
$$

we claim that

$$
\gamma(V)=\operatorname{gcd}\left(\left|H_{1}\right| t_{1}, \ldots,\left|H_{r}\right| t_{r}\right)
$$

Indeed, consider $\sigma \in V$. Note that, since $\sigma$ is a bijection, for every $j \in \llbracket r \rrbracket$, we have $\left|\sigma\left(H_{j}\right)\right|=\left|H_{j}\right|$, and thus, $\sigma$ maps an element $x \in H_{j}$ into an element belonging to $U_{j}=\bigcup_{\left|H_{i}\right|=\left|H_{j}\right|} H_{i}$. Thus, every $U_{j}$ is a union of $\sigma$-orbits and the distinct sets $U_{j}$ give a partition of $H$. Hence, the partition $T(\sigma)$ of $h$ induces a partition of 
$\left|U_{j}\right|=\left|H_{j}\right| t_{j}$ for all $j \in \llbracket r \rrbracket$. It follows that, for every $j \in \llbracket r \rrbracket \operatorname{gcd}(T(\sigma))|| U_{j} \mid$, and thus, $\operatorname{gcd}(T(\sigma))$ divides $\operatorname{gcd}\left(\left|U_{1}\right|, \ldots,\left|U_{r}\right|\right)$ for all $\sigma \in V$. Then, we also have that $\gamma(V)=\operatorname{lcm}\{\operatorname{gcd}(T(\sigma)): \sigma \in V\}$ divides $\operatorname{gcd}\left(\left|U_{1}\right|, \ldots,\left|U_{r}\right|\right)$. If $\operatorname{gcd}\left(\left|U_{1}\right|, \ldots,\left|U_{r}\right|\right)=1$, then we also have $\gamma(V)=1=\operatorname{gcd}\left(\left|U_{1}\right|, \ldots,\left|U_{r}\right|\right)$ and we have finished. Assume then that $\operatorname{gcd}\left(\left|U_{1}\right|, \ldots,\left|U_{r}\right|\right) \neq 1$. Then, in particular, $\left|U_{j}\right| \neq 1$ for all $j \in \llbracket r \rrbracket$ and it is meaningful to define a special cycle $\sigma_{j}$ on its elements in the following way. Let $H_{i_{1}}, \ldots H_{i_{t}}$ be the distinct sets of size $\left|H_{j}\right|=\ell \geq 1$ whose union is $U_{j}$ and order the elements inside each of them, say, $H_{i_{1}}=$ $\left\{h_{i_{1}}^{1}, \ldots, h_{i_{1}}^{\ell}\right\}, \ldots, H_{i_{t}}=\left\{h_{i_{t}}^{1}, \ldots, h_{i_{t}}^{\ell}\right\}$. Define $\sigma_{j}$ as the $\left|U_{j}\right|$-cycle on the elements of $U_{j}$ given by $\sigma_{j}=\left(h_{i_{1}}^{1} h_{i_{2}}^{1} \ldots h_{i_{t}}^{1} h_{i_{1}}^{2} h_{i_{2}}^{2} \ldots h_{i_{t}}^{2} \ldots h_{i_{1}}^{\ell} h_{i_{2}}^{\ell} \ldots h_{i_{t}}^{\ell}\right)$. It is immediately checked that $\sigma_{j}\left(H_{i_{k}}\right)=H_{i_{k+1}}$ for all $k \in \llbracket t-1 \rrbracket$ while $\sigma_{j}\left(H_{i_{t}}\right)=H_{i_{1}}$. Consider now $\sigma \in S_{h}$ defined by $\sigma=\sigma_{1} \cdots \sigma_{r}$. Then, $\sigma \in V$ and $T(\sigma)=\left[\left|U_{1}\right|, \ldots,\left|U_{r}\right|\right]$ and $\operatorname{gcd}(T(\sigma))=\operatorname{gcd}\left(\left|U_{1}\right|, \ldots,\left|U_{r}\right|\right)$ divides $\gamma(V)$. Thus, we obtain (17).

Thus, by (17), (15) and Theorem 20, we deduce that if

$$
\operatorname{gcd}\left(\left|H_{1}\right| t_{1}, \ldots,\left|H_{r}\right| t_{r}, \prod_{j=1}^{s}\left|N_{j}\right| !\right)=1
$$

then $C$ admits a $V$-anonymous and $W$-neutral resolute refinement. Moreover, by (17), (15) and Theorem 21, we deduce that if (18) holds true, not all the subclasses are singletons and one of the following conditions is satisfied:

(a) there exist at least two subclasses of odd size,

(b) $n \leq 3$,

(c) $k \in\{1, n-1\}$,

(d) $n$ is even and $k$ is odd,

then $C$ admits a $V$-anonymous, $W$-neutral and immune to the reversal bias resolute refinement.

\subsection{Alternatives as a subset of individuals}

Assume that the committee is facing the problem of electing a subcommittee of size $k$ among those members of the committee who are running for the selection. In this particular situation, we have that the alternatives to choose from are a subset of the set of individuals. Suppose that committee members also agree on the fact that the desired resolute refinement of $C$ should not distinguish among individuals who are candidates, should not distinguish among individuals who are not candidates and should be immune to the reversal bias.

Assume then $n \leq h$ and name $1, \ldots, n$ the individuals running for the selection. Thus, $N=\{1, \ldots, n\} \subseteq H$ is the set of alternatives to choose from and the procedure the committee is looking for corresponds to a $U$-consistent resolute refinement of $C$, where

$$
U=\{(\varphi, \psi, \rho) \in G: \forall i \in \llbracket n \rrbracket, \varphi(i)=\psi(i)\}
$$


Note that if $(\varphi, \psi, \rho) \in U$, then $\varphi(H \backslash N)=H \backslash N$. Note also that the above group $U$ is not necessarily regular. Consider, for instance, the case $h=4$ and $n=2$. Then, ((12)(34), (12), id) $\in U$ violates Theorem 12 in Bubboloni and Gori (2015). In the next proposition, we see that, as usual, a suitable coprimality condition is equivalent to regularity.

Proposition 22 Let $n \leq h$. The group

$$
U=\{(\varphi, \psi, \rho) \in G: \forall i \in \llbracket n \rrbracket, \varphi(i)=\psi(i)\}
$$

is regular if and only if $\operatorname{gcd}(h, n)=1$.

Proof We first describe in a convenient way the elements in $U$. Let $(\varphi, \psi, \rho) \in U$ and let

$$
\psi=\prod_{i=1}^{s} \gamma_{i},
$$

where the $\gamma_{j}$ are disjoint cycles, constituents of $\psi$ and $s \geq 0$. The case $s=0$ is to be intended as taking the product over the empty set, and by definition is equal to id. Since $\psi \in S_{n}$ is a permutation of the set $N \subseteq H$ and $\varphi \in S_{h}$ is a permutation of the set $H$ sharing with $\psi$ the same behavior on $N$, we have that $\varphi=\psi v$ for some permutation $v$ of the set $H \backslash N$. Thus, there exist $r \geq 0$ disjoint cycles $\gamma_{j}$ on the set $H \backslash N$, with $j \in\{s+1, \ldots, s+r\}$, such that

$$
\varphi=\prod_{i=1}^{s+r} \gamma_{i}
$$

and the above writing expresses $\varphi$ as product of its constituents. Note that if $r=0$, the set $\{s+1, \ldots, s+r\}$ is empty and no cycle has to be added.

We claim that the following property holds true:

$$
\text { if }(\varphi, \psi, \rho) \in U \text {, then } \operatorname{gcd}(T(\varphi))|\operatorname{gcd}(h, \operatorname{gcd}(T(\psi)))| \operatorname{gcd}(h, n) \text {. }
$$

Indeed, let $(\varphi, \psi, \rho) \in U$. For $\psi$, we have the representation (20) with $s \geq 0$, and for $\varphi$ the representation (21) with $r \geq 0$. If $\psi=\mathrm{id} \in S_{n}$, then $\varphi$ admits at least one fixed point. Thus $\operatorname{gcd}(T(\psi))=1$ and $\operatorname{gcd}(T(\varphi))=1$ and (22) is obvious. If $\psi \neq$ id, we have $s \geq 1$ and

$$
\left.\operatorname{gcd}(T(\varphi)) \mid \operatorname{gcd}\left(\left|\gamma_{i}\right|\right)\right)_{i=1}^{s}=\operatorname{gcd}(T(\psi)) \mid n
$$

On the other hand, $\operatorname{gcd}(T(\varphi)) \mid h$ and thus (22) follows. Assume now that $\operatorname{gcd}(h, n)=$ 1 . Then, by (22), we have $\operatorname{gcd}(T(\varphi))=1$. In order to show that $U$ is regular, we show that the conditions (a) and (b) in Theorem 12 in Bubboloni and Gori (2015) are satisfied. Let $(\varphi, \psi$, id $) \in U$, with $\psi \neq \mathrm{id}$, and $\pi$ be a prime such that $|\psi|_{\pi}=\pi^{a}$, for some $a \in \mathbb{N}$. Then, obviously $\pi^{a} \nmid \operatorname{gcd}(T(\varphi))=1$. 
Let next $\left(\varphi, \psi, \rho_{0}\right) \in U$, with $\psi^{2}=$ id and $\psi$ not a conjugate $\rho_{0}$. Then, clearly $2 \nmid \operatorname{gcd}(T(\varphi))=1$. Assume now that $\operatorname{gcd}(h, n) \neq 1$. Let $\pi$ be prime dividing $\operatorname{gcd}(h, n)$. Let $\psi \in S_{n}$ be a product of $n / \pi$ disjoint $\pi$-cycles and $\varphi \in S_{h}$ be a product of $h / \pi$ disjoint $\pi$-cycles with the first $n / \pi$ of them equal to those forming $\psi$. Then, $(\varphi, \psi$, id $) \in U$ and $|\psi|_{\pi}=\pi \mid \operatorname{gcd}(T(\varphi))=\pi$. Thus, condition (a) in Theorem 12 in Bubboloni and Gori (2015) is not satisfied. Hence, $U$ is not regular.

Note that the arithmetical conditions $n \leq h, \operatorname{gcd}(h, n)=1$ are surely milder than the condition $\operatorname{gcd}(h, n !)=1$ which guarantees the existence of a resolute, anonymous and neutral SPC. For instance, when $h=4, n=3$ the first conditions are satisfied while the second is not.

By Theorem 10, we deduce that if $\operatorname{gcd}(h, n)=1$ and one of the following conditions is satisfied:

(b) $n \leq 3$,

(c) $k \in\{1, n-1\}$,

(d) $n$ is even and $k$ is odd,

then $C$ admits a $U$-consistent resolute refinement. ${ }^{15}$

Observe that the nature of the group $U$ defined by (19) is very different from the groups of symmetry considered in Sects. 8.1 and 8.2. In fact, $U$ cannot be expressed as a direct product of subgroups in $S_{h}$ and $S_{n}$. Indeed, assume by contradiction that $U=V \times W \times \Omega$, for suitable $V \leq S_{h}$ and $W \leq S_{n}$. Then, for every $(\varphi, \psi, \rho) \in U$ we also have (id, $\psi, \rho) \in U$. But picking $(\varphi, \psi, \rho) \in U$, with $\psi \neq$ id, we instead necessarily have $\varphi \neq$ id and thus (id, $\psi, \rho) \notin U$.

The fact that different typologies of subgroups of $G$ could be of interest in the applications make our theoretical approach much more concrete than one could in principle imagine.

\subsection{Committee members with diverse decision power}

Assume that committee members have different decision power described by an order on the set of individuals. Orders model those situations in which it is reasonable to divide individuals into disjoint groups where all individuals have the same decision power and to rank such groups. Consider, for instance, a faculty committee composed by full and associate professors which is going to select some applicants for an academic position. There are in this case two well distinguished groups in the committee and a natural hierarchy between them ranking first the full professors and second the associate professors. Assume then that committee members agree to use a resolute refinement of $C$ consistent with the decision power of individuals and which equally treat all the alternatives.

Consider then the relation $R \in \mathbf{O}(H)$ defined as follows: For every $x, y \in H$, we define $x \succeq_{R} y$ if and only if individual $x$ has a decision power which is at least as great as the one of individual $y$. The procedure the committee is looking for is a resolute refinement of $C$ which is $\operatorname{Aut}(R)$-anonymous and neutral, where $\operatorname{Aut}(R)$ is the group

\footnotetext{
$\overline{15}$ Note that condition (a) in Theorem 10 cannot be satisfied because $\left(\varphi, \rho_{0}, \rho_{0}\right) \in U$, for any $\varphi \in S_{h}$ such that $\varphi(i)=\rho_{0}(i)$ for all $i \in \llbracket n \rrbracket$.
} 
$\operatorname{Aut}(R)=\left\{\sigma \in S_{h}: \forall x, y \in H, \sigma(x) \succeq_{R} \sigma(y)\right.$ if and only if $\left.x \succeq_{R} y\right\}$,

called the group of automorphisms of $R$.

It is well known that the relation

$$
I(R)=\left\{(x, y) \in H^{2}:(x, y) \in R \text { and }(y, x) \in R\right\}
$$

is an equivalence relation in $H$. Denote by $H_{1}, \ldots, H_{r}$, where $r \geq 1$, the equivalence classes of $I(R)$. We claim that

$$
\operatorname{Aut}(R)=\left\{\varphi \in S_{h}: \forall j \in \llbracket r \rrbracket, \varphi\left(H_{j}\right)=H_{j}\right\}
$$

Indeed, first order the sets $H_{1}, \ldots, H_{r}$ so that $x \in H_{i}$ and $y \in H_{j}$ with $i<j$ if and only if $x \succ_{R} y$. The permutations in $K=\left\{\varphi \in S_{h}: \forall j \in \llbracket r \rrbracket, \varphi\left(H_{j}\right)=H_{j}\right\}$ clearly form a subgroup of $S_{h}$. We show that $K \leq \operatorname{Aut}(R)$. Pick $\varphi \in K$ and let $x, y \in H$ with $x \succeq_{R} y$. If we also have $y \succeq_{R} x$, then $x$ and $y$ belong to the same $H_{j}$ and thus, by definition of $K, \varphi(x)$ and $\varphi(y)$ belong to $H_{j}$ too. Thus $\varphi(x) \succeq_{R} \varphi(y)$. If instead $y \nsucceq_{R} x$, then $x \succ_{R} \quad y$. Thus, we have $x \in H_{i}$ and $y \in H_{j}$ for some $i, j \in \llbracket r \rrbracket$ with $i<j$. Thus, we have $\varphi(x) \in \varphi\left(H_{i}\right)=H_{i}$ and $\varphi(y) \in \varphi\left(H_{j}\right)=H_{j}$ so that, $\varphi(x) \succ_{R} \varphi(y)$ and then, in particular, $\varphi(x) \succeq_{R} \varphi(y)$. Let now $x, y \in H$ with $\varphi(x) \succeq_{R} \varphi(y)$. Then, the same argument applies to $\varphi^{-1}$, giving $x \succeq_{R} y$.

We next show that $\operatorname{Aut}(R) \leq K$. Let $\varphi \in \operatorname{Aut}(R)$ and $j \in \llbracket r \rrbracket$. We need to see that $\varphi\left(H_{j}\right)=H_{j}$. To that purpose, since $\varphi$ is a bijection, it is enough to show that $\varphi\left(H_{j}\right) \subseteq H_{j}$. Pick $x \in H_{j}$. Since $H_{1}, \ldots, H_{r}$ are a partition of $H$, there exists $i \in \llbracket r \rrbracket$, such that $y=\varphi(x) \in H_{i}$. Assume, by contradiction, that $i \neq j$. Then, we have that $x \succ_{R} y$ or $y \succ_{R} x$. Assume that $x \succ_{R} y=\varphi(x)$. Since $\varphi \in \operatorname{Aut}(R)$, applying $\varphi$ to that relation, we get $\varphi(x) \succ_{R} \varphi^{2}(x)$, and thus, by transitivity $x \succ_{R} \varphi^{2}(x)$. Iterating this argument, we then find $x \succ_{R} \varphi^{m}(x)$, for every $m \in \mathbb{N}$. Considering now $m$ such that $\varphi^{m}(x)=x$, we get the contradiction $x \succ_{R} x$. A similar argument shows the impossibility of $y \succ_{R} x$.

As a consequence, by $(14), \gamma(\operatorname{Aut}(R))=\operatorname{gcd}\left(\left|H_{1}\right|, \ldots,\left|H_{r}\right|\right)$. Thus, by Theorem 20 , we deduce that if

$$
\operatorname{gcd}\left(\left|H_{1}\right|, \ldots,\left|H_{r}\right|,|N| !\right)=1,
$$

then $C$ admits an $\operatorname{Aut}(R)$-anonymous, neutral and resolute refinement. Moreover, by Theorem 21, we deduce that if (24) holds true and one of the following conditions is satisfied:

(b) $n \leq 3$,

(c) $k \in\{1, n-1\}$,

(d) $n$ is even and $k$ is odd,

then $C$ admits an $\operatorname{Aut}(R)$-anonymous, neutral and immune to the reversal bias resolute refinement. $^{16}$

16 Note that condition (a) in Theorem 21 cannot be satisfied because obviously $S_{n}$ contains permutations which are conjugate of $\rho_{0}$. 
Assume now that the conditions for the existence of $\operatorname{Aut}(R)$-anonymous and neutral resolute refinements of $C$ are satisfied. In general, such refinements of $C$ are more than one. However, the information we get from $R$ goes further the mere knowledge of the indifference sets of $R$ and we can use this information for better selecting the resolute refinement. In order to explain this point, assume that the committee has a president, say individual 1, and assume that the president has a decision power which is greater than the one of all the other members of the committee. Assume further that all individuals but the president have the same decision power. Such a situation can be modeled by the order $R \in \mathbf{O}(H)$ defined by

$$
R=\left\{(1, y) \in H^{2}: y \in H\right\} \cup\left\{(x, y) \in H^{2}: x, y \in H \backslash\{1\}\right\} .
$$

In this case, the indifference classes of $I(R)$ are $H_{1}=\{1\}$ and $H_{2}=\{2, \ldots, h\}$ so that (24) holds and $C$ admits a resolute refinement which is $\operatorname{Aut}(R)$-anonymous and neutral. Note that

$$
\operatorname{Aut}(R)=\left\{\varphi \in S_{h}: \varphi(1)=1\right\} .
$$

As already emphasized, resolute refinements can be many and the problem of selecting one of them is certainly crucial. The special characteristics of the situation under consideration can be used to make this selection. Assume that $k=1$. Then, among those resolute refinements it is natural to choose the one obtained by letting the president break the ties by choosing his/her best alternative. This is exactly the classic tie-breaker method widely used in practical situations. Another $\operatorname{Aut}(R)$-anonymous and neutral resolute refinement can be obtained, for instance, by selecting the alternative which is the worst one for the president. Of course, this option is not in line with the decision power among individuals and the role of the president, and it is reasonably discarded. Anyway it is important to observe that it arises as one of the possible $\operatorname{Aut}(R)$-anonymous and neutral resolute refinements.

Assuming now $k \geq 2$, there is no standard and intuitive way to select a resolute refinement. However, looking at the particular characteristics of the decision problem some resolute refinements can be easily discarded and others can be reasonably taken into consideration.

Next consider a planner who wants to organize a committee made up of $h$ members to judge some candidates applying for $k$ job positions, using some well-established $k$ SCC $C$. The planner reasonably desires to dispose of a resolute refinement of $C$ in order to produce an actual decision. Preserving neutrality, he can get that goal by renouncing the full anonymity in the collective decision. To that purpose, he can simply fix two numbers $h_{1}$ and $h_{2}$ with $\operatorname{gcd}\left(h_{1}, h_{2}\right)=1$ and $h_{1}+h_{2}=h$, split the committee into two subcommittees $H_{1}$ and $H_{2}$ with $\left|H_{1}\right|=h_{1}$ and $\left|H_{2}\right|=h_{2}$, and let the decision power relation among the committee members be described by a suitable $R \in \mathbf{O}(H)$ with indifference sets $H_{1}$ and $H_{2}$. The planner can generally choose the most suitable partition $\left[h_{1}, h_{2}\right]$ among many. For instance, if $h=7$, he can choose $\left[h_{1}, h_{2}\right]$ among $[1,6],[2,5]$ and $[3,4]$; if $h=8$, he can choose $\left[h_{1}, h_{2}\right]$ between $[1,7]$ and $[3,5]$. Note that those choices work whatever the number $n$ of candidates could be. Knowing the number $n$ of candidates can lead to other possibilities. For instance, if $h=7$ 
and $n=4$, we have $\operatorname{gcd}(h, n !)=1$, and thus, the planner is not obliged to split the committee to reach the goal. In that case, he can reach full anonymity.

\subsection{Final considerations}

The applications described in this section indicate some strategies to identify, in a decision process, the group $U \leq S_{h} \times S_{n} \times \Omega$ consistent with the specific situation under examination. The group $U$ is in no way arbitrary but must reflect the typology of the committee called to make a decision and the aim of the decision itself. Once $U$ is determined, our theorems establish whether $U$-consistent $(U$-symmetric) resolute refinements of a given $k$-SCC (SPC) exist. When such resolute refinements exist, their use strengthen the social acceptability of the decision and sounds as a basic and necessary fulfillment of fairness.

\section{Building tie breaking rules}

Up to now, we mainly focused on the existence of resolute refinements of given SPCs or $k$-SCCs with special properties. All the results stated in the previous sections originated, however, by quite technical results that allow in fact to get a complete information about all the possible refinements (especially Propositions 27, 30 and 32 and Theorems 28, 31 and 33 in "Appendix B"). More precisely, they allow to potentially build and count all the resolute refinements having the desired properties and give rise to a precise algorithm which we are going to make explicit in this section.

\subsection{The general construction}

Let $C$ be a decisive SPC or a decisive $k$-SCC and let $U$ be a regular subgroup of $G$. Due to the fact that the group $U$ acts on the set $\mathcal{P}$ of preference profiles (Bubboloni and Gori 2015, Proposition 2; see also Appendix A), we have that the set of preference profiles can be partitioned into subsets called $U$-orbits. Each $U$-orbit is obtained by considering all the preference profiles of the type $p^{(\varphi, \psi, \rho)}$, where $p$ is fixed and $(\varphi, \psi, \rho)$ varies on $U$. Picking an element from each $U$-orbit, we build what is called a system of representatives of the $U$-orbits. Note that the concept of system of representatives has nothing to do with $C$ but only depends on the set $\mathcal{P}$ and the group $U$ ("Appendix B.1"). Of course, finding explicitly a system of representatives is in general a complex problem. However, that can be managed for small values of $h$ and $n$, that is, when the size $n !^{h}$ of $\mathcal{P}$ is not too large.

We explain now how the systems of representatives turn out to be fundamental for building the desired resolute refinements of $C$.

Case 1. Assume that $C$ is $U$-consistent and $U \leq S_{h} \times S_{n} \times\{\mathrm{id}\}$. Thus, by Theorem 9 or Corollary 11, we know that $C$ admits a $U$-consistent resolute refinement. Once a system of representatives $\left(p^{j}\right)_{j=1}^{m}$ is found, a $U$-consistent resolute refinement $f$ of $C$ can be build as follows: 
(1) for every $j \in\{1, \ldots, m\}$, pick $x_{j} \in C\left(p^{j}\right),{ }^{17}$

(2) for every $p \in \mathcal{P}$, set $f(p)=\left\{\psi x_{j}\right\}$, where $j \in\{1, \ldots, m\}$ and $(\varphi, \psi$, id $) \in U$ are such that $p=p^{j(\varphi, \psi, \text { id })}$.

By Proposition 30 and its proof and Theorem 31, $f$ is well defined and all the $U$ consistent resolute refinements of $C$ can be built in this way.

Case 2. Assume that $C$ is $U$-consistent and $U \not \leq S_{h} \times S_{n} \times\{\mathrm{id}\}$ (if $C$ is a $k$ SCC also assume that one of the conditions (a), (b), (c) and (d) of Theorem 10 is satisfied). Thus, by Theorem 9 or Theorem 10, we know that $C$ admits a $U$-consistent resolute refinement. This time to explicitly construct those refinements, once a system of representatives $\left(p^{j}\right)_{j=1}^{m}$ is found, we have to divide the set of indexes $\{1, \ldots, m\}$ in two sets. The first one, denoted by $\mathcal{P}_{1}^{U}$, is the set of indexes $j$ such that if there exists $(\varphi, \psi, \rho) \in U$ such that $p^{j}=p^{j(\varphi, \psi, \rho)}$, then necessarily $\rho=\mathrm{id}$; the second one, denoted by $\mathcal{P}_{2}^{U}$, is the set containing the other indexes (one of these sets might be empty). ${ }^{18}$ By the regularity of $U$, for every $j \in \mathcal{P}_{2}^{U}$, there exists (a unique) $\psi_{j} \in S_{n}$ such that if $p^{j}=p^{j\left(\varphi, \psi, \rho_{0}\right)}$ for a certain $\left(\varphi, \psi, \rho_{0}\right) \in U$, then necessarily $\psi=\psi_{j}$. Moreover, for every $j \in \mathcal{P}_{2}^{U}$, there exists $\sigma_{j} \in S_{n}$ such that $\psi_{j}=\sigma_{j} \rho_{0} \sigma_{j}^{-1}$.

Fixed now an element $\left(\varphi_{*}, \psi_{*}, \rho_{0}\right) \in U$, compute, for every $j \in \mathcal{P}_{1}^{C}$, the set

$$
A_{1}^{C}\left(p^{j}\right)=\left\{(y, z) \in C\left(p^{j}\right) \times C\left(p^{j\left(\varphi_{*}, \psi_{*}, \rho_{0}\right)}\right): y \neq z\right\}
$$

and, for every $j \in \mathcal{P}_{2}^{C}$, the set

$$
A_{2}^{C}\left(p^{j}\right)=\left\{x \in C\left(p^{j}\right): \psi_{j} x \neq x\right\} .
$$

Thus, a $U$-consistent resolute refinement $f$ of $C$ can be built as follows:

(1) for every $j \in \mathcal{P}_{1}^{U}$, pick $\left(y_{j}, z_{j}\right) \in A_{1}^{C}\left(p^{j}\right)$;

(2) for every $j \in \mathcal{P}_{2}^{U}$, pick $x_{j} \in A_{2}^{C}\left(p^{j}\right)$

(3) for every $p \in \mathcal{P}$,

(i) if there exists $j \in \mathcal{P}_{1}^{U}$ and $(\varphi, \psi$, id $) \in U$ such that $p=p^{j(\varphi, \psi, \text { id })}$, then $f(p)=\left\{\psi y_{j}\right\}$

(ii) if there exists $j \in \mathcal{P}_{1}^{U}$ and $\left(\varphi, \psi, \rho_{0}\right) \in U$ such that $p=p^{j\left(\varphi, \psi, \rho_{0}\right)}$, then $f(p)=\left\{\psi \psi_{*}^{-1} z_{j}\right\}$

(iii) if there exists $j \in \mathcal{P}_{2}^{U}$ and $(\varphi, \psi, \rho) \in U$ such that $p=p^{j(\varphi, \psi, \rho)}$, then $f(p)=\left\{\psi \sigma_{j} \rho \sigma_{j}^{-1} x_{j}\right\}$.

By Proposition 32 and its proof and Theorem 33, $f$ is well defined and all the $U$ consistent resolute refinements of $C$ can be built in this way.

Case 3. Assume that $C$ is a $U$-symmetric SPC and assume that one of the conditions (ii) and (iii) of Theorem 7 is satisfied. Thus, by Theorem 7, we know that $C$ admits a $U$-symmetric resolute refinement. In order to build a refinement, we do not need now to distinguish the two cases $U \leq S_{h} \times S_{n} \times\{\mathrm{id}\}$ and $U \not \leq S_{h} \times S_{n} \times\{\mathrm{id}\}$. Indeed,

${ }^{17}$ Recall that $x_{j}$ is a linear order when $C$ is a SPC and a set of size $k$ when $C$ is a k-SCC.

18 See "Appendix B.1". 
given a system of representatives $\left(p^{j}\right)_{j=1}^{m}$, a $U$-symmetric resolute refinement $f$ of $C$ can be build as follows:

(1) for every $j \in\{1, \ldots, m\}$, pick $q_{j} \in C\left(p^{j}\right) \cap S\left(p^{j}\right)$, where

$$
S\left(p^{j}\right)=\left\{q \in \mathbf{L}(N): \forall(\varphi, \psi, \rho) \in U, \quad \text { if } p^{j(\varphi, \psi, \rho)}=p^{j} \quad \text { then } \quad \psi q \rho=q\right\},
$$

(2) for every $p \in \mathcal{P}$, set $f(p)=\left\{\psi q_{j}\right\}$, where $j \in\{1, \ldots, m\}$ and $(\varphi, \psi$, id $) \in U$ are such that $p=p^{j(\varphi, \psi, \text { id })}$.

By Proposition 27 and its proof and Theorem 28, $f$ is well defined and all the $U$ symmetric resolute refinements of $C$ can be built in this way.

\subsection{A concrete example}

Let $h=5$ and $n=3$. Thus $N=\{1,2,3\}$ and, by Theorem $16, G$ is regular, so that we can choose $U=G$. Note also that $\rho_{0}=(13)$. Consider now the Borda 2-SCCs denoted by $\mathrm{BOR}_{2}$ and defined, for every $p \in \mathcal{P}$, by

$$
\operatorname{BOR}_{2}(p)=\left\{W \subseteq N:|W|=2, \forall x \in N, y \in N \backslash W s_{p}^{\text {bor }}(x) \geq s_{p}^{\text {bor }}(y)\right\},
$$

where, $s_{p}^{\text {bor }}: N \rightarrow \mathbb{R}$ is the well-known Borda score function associated with $p$. Note that $\mathrm{BOR}_{2}$ is decisive and $G$-consistent. However, $\mathrm{BOR}_{2}$ is not resolute.

Since condition (b) of Theorem 10 is satisfied, we get that $\mathrm{BOR}_{2}$ admits a $G$ consistent resolute refinement. Let us apply then what described in Case 2 of Sect. 9.1.

A system of representatives of the $G$-orbits for this particular situation is available in Bubboloni and Gori (2015, p. 83). We refer here to that system and use the same notation. As proved in Bubboloni and Gori (2016b), we have

$$
\begin{aligned}
& \mathcal{P}_{1}^{G}=\{2,4,5,7,8,10,11,12,14,15,16,18,21,22,23,24\} \text { and } \\
& \mathcal{P}_{2}^{G}=\{1,3,6,9,13,17,19,20,25,26\} .
\end{aligned}
$$

Choose $\left(\varphi_{*}, \psi_{*}, \rho_{0}\right)=$ (id, id, $\left.\rho_{0}\right)$, the simplest element in $G$ having a not trivial third component. Then, an easy computation, carried out just using the definition of $A_{1}^{\mathrm{BOR}_{2}}\left(p^{j}\right)$ and $A_{2}^{\mathrm{BOR}_{2}}\left(p^{j}\right)$, leads to Tables 2 and 3 . In order to let the reader be aware of the work done, we comment in detail a couple of interesting cases. Consider first

$$
p^{10}=\left[\begin{array}{lllll}
1 & 1 & 1 & 2 & 2 \\
2 & 2 & 2 & 1 & 3 \\
3 & 3 & 3 & 3 & 1
\end{array}\right]
$$

and note that $10 \in \mathcal{P}_{1}^{G}$. We have $s_{p^{10}}^{\text {bor }}(1)=s_{p^{10}}^{\text {bor }}(2)=7$ and $s_{p^{10}}^{\text {bor }}(3)=1$. Thus,

$$
\operatorname{BOR}_{2}\left(p^{10}\right)=\{\{1,2\}\} \text { and } \operatorname{BOR}_{2}\left(p^{10\left(\mathrm{id}, \mathrm{id}, \rho_{0}\right)}\right)=\{\{1,3\},\{2,3\}\}
$$


Table 2 Computation of $A_{1}^{\mathrm{BOR}_{2}}\left(p^{j}\right)$ for $j \in \mathcal{P}_{1}^{G}$

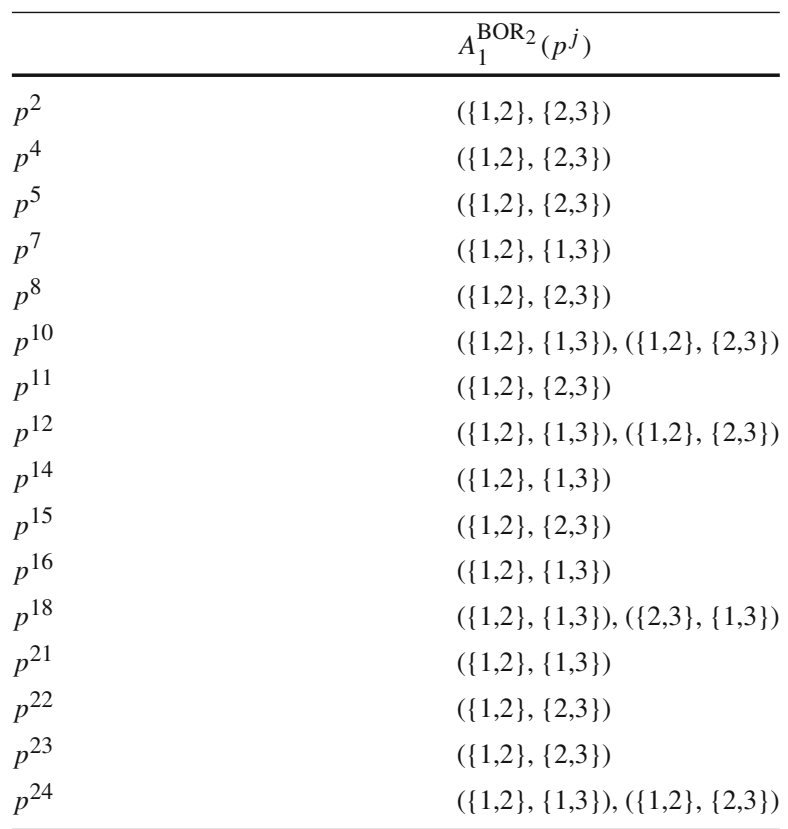

Since $\{1,2\} \neq\{1,3\}$ and $\{1,2\} \neq\{2,3\}$, we then get

$$
A_{1}^{\mathrm{BOR}_{2}}\left(p^{10}\right)=\{(\{1,2\},\{1,3\}),(\{1,2\},\{2,3\})\} .
$$

\section{Consider now}

$$
p^{25}=\left[\begin{array}{lllll}
1 & 1 & 3 & 2 & 3 \\
2 & 2 & 2 & 3 & 1 \\
3 & 3 & 1 & 1 & 2
\end{array}\right]
$$

and note that $25 \in \mathcal{P}_{2}^{G}$ and $p^{25}=p^{25\left((45),(13), \rho_{0}\right)}$. Thus, $\psi_{25}=$ (13). Moreover, $s_{p^{25}}^{\text {bor }}(1)=s_{p^{25}}^{\text {bor }}(2)=s_{p^{25}}^{\text {bor }}(3)=5$ so that

$$
\operatorname{BOR}_{2}\left(p^{25}\right)=\{\{1,2\},\{1,3\},\{2,3\}\} .
$$

In order to compute $A_{2}^{\mathrm{BOR}_{2}}\left(p^{25}\right)$, we need to exclude the sets in $\mathrm{BOR}_{2}\left(p^{25}\right)$ which are fixed by the permutation $\psi_{25}$, and thus, we get

$$
A_{2}^{\mathrm{BOR}_{2}}\left(p^{25}\right)=\{\{1,2\},\{2,3\}\}
$$

Looking at Tables 2 and 3, we can understand, in particular, that in the case of 5 individuals and 3 alternatives there exists $2^{5}$ anonymous, neutral and immune to the reversal bias resolute refinements of $\mathrm{BOR}_{2}$. In order to select one of them, one just 
Table 3 Computation of $A_{2}^{\mathrm{BOR}_{2}}\left(p^{j}\right)$ for $j \in \mathcal{P}_{2}^{G}$

\begin{tabular}{ll}
\hline & $A_{2}^{\mathrm{BOR}_{2}}\left(p^{j}\right)$ \\
\hline$p^{1}$ & $\{1,2\}$ \\
$p^{3}$ & $\{1,2\}$ \\
$p^{6}$ & $\{1,2\}$ \\
$p^{9}$ & $\{1,2\}$ \\
$p^{13}$ & $\{1,2\}$ \\
$p^{17}$ & $\{1,2\}$ \\
$p^{19}$ & $\{1,3\}$ \\
$p^{20}$ & $\{1,2\}$ \\
$p^{25}$ & $\{1,2\},\{2,3\}$ \\
$p^{26}$ & $\{1,2\}$ \\
\hline
\end{tabular}

Table 4 Particular breaking tie

\begin{tabular}{lll}
\hline & $f\left(p^{j}\right)$ & $f\left(p^{j\left(\mathrm{id}, \mathrm{id}, \rho_{0}\right)}\right)$ \\
\hline$p^{10}$ & $\{1,2\}$ & $\{2,3\}$ \\
$p^{12}$ & $\{1,2\}$ & $\{2,3\}$ \\
$p^{18}$ & $\{2,3\}$ & $\{1,3\}$ \\
$p^{24}$ & $\{1,2\}$ & $\{2,3\}$ \\
$p^{25}$ & $\{2,3\}$ & \\
\hline
\end{tabular}

needs to look at the preference profiles $p^{10}, p^{12}, p^{18}, p^{24}, p^{25}$ and ask which of the two possibilities in the tables is the one that best fits the decision problem for which the refinement of the Borda $2-\mathrm{SCC}$ is required. ${ }^{19}$ Once this choice is made, one can write down automatically the values $f(p)$ for all $p \in \mathcal{P}$.

Assume, for instance, that the refinement $f$ identified by the choices expressed in Table 4 is chosen. Let us compute then $f(p)$, where

$$
p=\left[\begin{array}{lllll}
2 & 1 & 3 & 1 & 1 \\
1 & 3 & 2 & 3 & 2 \\
3 & 2 & 1 & 2 & 3
\end{array}\right]
$$

We have that $p=p^{24\left(\varphi, \psi, \rho_{0}\right)}$, where $\varphi=(14)(35)$ and $\psi=$ (123). Notice that $24 \in \mathcal{P}_{1}^{G}$ so that, according to Case 2 discussed in Sect. 9.1, we get

$$
f(p)=\left\{\psi \psi_{*}^{-1} z_{j}\right\}=\{(123) \operatorname{id}\{2,3\}\}=\{\{1,3\}\} .
$$

${ }_{19}$ In Table 2, if $(y, z)$ appears in $A_{1}^{\mathrm{BOR}_{2}}\left(p^{j}\right)$, then we have a resolute refinement $f$ with $f\left(p^{j}\right)=y$ and $f\left(p^{j\left(\mathrm{id}, \mathrm{id}, \rho_{0}\right)}\right)=z$; in Table 3, if $x$ appears in $A_{2}^{\mathrm{BOR}_{2}}\left(p^{j}\right)$, then we have a resolute refinement $f$ with $f\left(p^{j}\right)=x$. Recall that in this last case, the value of $f\left(p^{j\left(\mathrm{id}, \mathrm{id}, \rho_{0}\right)}\right)$ is induced from the choice for $f\left(p^{j}\right)$. 
Acknowledgements Open access funding provided by Università degli Studi di Firenze within the CRUICARE Agreement.

Open Access This article is licensed under a Creative Commons Attribution 4.0 International License, which permits use, sharing, adaptation, distribution and reproduction in any medium or format, as long as you give appropriate credit to the original author(s) and the source, provide a link to the Creative Commons licence, and indicate if changes were made. The images or other third party material in this article are included in the article's Creative Commons licence, unless indicated otherwise in a credit line to the material. If material is not included in the article's Creative Commons licence and your intended use is not permitted by statutory regulation or exceeds the permitted use, you will need to obtain permission directly from the copyright holder. To view a copy of this licence, visit http://creativecommons.org/licenses/by/4.0/.

\section{Appendix}

\section{A Symmetry and consistency}

First of all, let us note that, for every $\sigma \in S_{n}, \rho \in \Omega, W_{1}, W_{2} \in \mathbb{P}(N), \mathbb{W}_{1}, \mathbb{W}_{2} \subseteq$ $\mathbb{P}(N), R_{1}, R_{2} \in \mathbf{R}(N)$ and $\mathbf{Q}_{1}, \mathbf{Q}_{2} \subseteq \mathbf{R}(N)$, we have that

$$
\begin{aligned}
& \sigma W_{1} \subseteq \sigma W_{2} \Leftrightarrow W_{1} \subseteq W_{2}, \quad \sigma \mathbb{W}_{1} \subseteq \sigma \mathbb{W}_{2} \Leftrightarrow \mathbb{W}_{1} \subseteq \mathbb{W}_{2}, \\
& R_{1} \subseteq R_{2} \Leftrightarrow \sigma R_{1} \subseteq \sigma R_{2} \Leftrightarrow R_{1} \rho \subseteq R_{2} \rho \\
& \mathbf{Q}_{1} \subseteq \mathbf{Q}_{2} \Leftrightarrow \sigma \mathbf{Q}_{1} \subseteq \sigma \mathbf{Q}_{2} \Leftrightarrow \mathbf{Q}_{1} \rho \subseteq \mathbf{Q}_{2} \rho .
\end{aligned}
$$

From those inclusions, analogous relations for equalities hold true. We freely use those properties in the rest of the paper. We emphasize their use when particularly remarkable.

Let $U \leq G$. In Bubboloni and Gori (2015, Proposition 2), it is shown that the definition (6) determines an action of $U$ on the set of preference profiles. In particular, for every $p \in \mathcal{P}$ and $\left(\varphi_{1}, \psi_{1}, \rho_{1}\right),\left(\varphi_{2}, \psi_{2}, \rho_{2}\right) \in U$, we have

$$
p^{\left(\varphi_{1} \varphi_{2}, \psi_{1} \psi_{2}, \rho_{1} \rho_{2}\right)}=\left(p^{\left(\varphi_{2}, \psi_{2}, \rho_{2}\right)}\right)^{\left(\varphi_{1}, \psi_{1}, \rho_{1}\right)}
$$

The above equality is a main tool throughout the paper. To begin with, it allows to prove the basic results on symmetry and consistency stated in Sect. 4.

Proof of Proposition 1 (i) Let $C \in \mathfrak{P}^{* U}$ and $p \in \mathcal{P}$. Consider first $(\varphi, \psi$, id) $\in U$. Since $C$ is $U$-symmetric, then $C\left(p^{(\varphi, \psi, \text { id })}\right)=\psi C(p)$ id $=\psi C(p)$ and $(8)$ is satisfied. In order to prove (9) assume, by contradiction, that $|C(p)|=1$ and that there exists $\left(\varphi, \psi, \rho_{0}\right) \in G$ such that $C\left(p^{\left(\varphi, \psi, \rho_{0}\right)}\right)=\psi C(p)$. Then, $C(p)=\{q\}$ for some $q \in \mathbf{L}(N)$ and, by the $U$-symmetry of $C$, we get $\psi C(p) \rho_{0}=\psi C(p)$. Thus, $C(p) \rho_{0}=C(p)$, that is, $q \rho_{0}=q$, which gives the contradiction $\rho_{0}=\mathrm{id}$.

(ii) From (i) we know that $\mathfrak{P}^{* U} \subseteq \mathfrak{P}^{U}$. On the other hand, if $C \in \mathfrak{P}^{U}$ we have that condition (8) is satisfied and, since $U \leq S_{h} \times S_{n} \times\{\mathrm{id}\}$, that equals condition (7) so that $C \in \mathfrak{P}^{* U}$. 
Proof of Proposition 2 The fact that $\mathfrak{P}^{\left\langle U_{1}, U_{2}\right\rangle} \subseteq \mathfrak{P}^{* U_{1}} \cap \mathfrak{P}^{* U_{2}}$ is obvious. We show the other inclusion. Let $C \in \mathfrak{P}^{* U_{1}} \cap \mathfrak{P}^{* U_{2}}$. Consider the set

$$
W=\left\{(\varphi, \psi, \rho) \in G: \forall p \in \mathcal{P}, C\left(p^{(\varphi, \psi, \rho)}\right)=\psi C(p) \rho\right\} .
$$

We show that $W$ is a subgroup of $G$. Let $\left(\varphi_{1}, \psi_{1}, \rho_{1}\right),\left(\varphi_{2}, \psi_{2}, \rho_{2}\right) \in W$ and show that $\left(\varphi_{1} \varphi_{2}, \psi_{1} \psi_{2}, \rho_{1} \rho_{2}\right) \in W$. Given $p \in \mathcal{P}$, by (26) and recalling that $\Omega$ is abelian, we have

$$
\begin{aligned}
C\left(p^{\left(\varphi_{1} \varphi_{2}, \psi_{1} \psi_{2}, \rho_{1} \rho_{2}\right)}\right) & =C\left(\left(p^{\left(\varphi_{2}, \psi_{2}, \rho_{2}\right)}\right)^{\left(\varphi_{1}, \psi_{1}, \rho_{1}\right)}\right)=\psi_{1} C\left(p^{\left(\varphi_{2}, \psi_{2}, \rho_{2}\right)}\right) \rho_{1} \\
& =\psi_{1} \psi_{2} C(p) \rho_{2} \rho_{1}=\psi_{1} \psi_{2} C(p) \rho_{1} \rho_{2} .
\end{aligned}
$$

Since $W$ is a group and contains both $U_{1}$ and $U_{2}$, then we necessarily have $W \geq$ $\left\langle U_{1}, U_{2}\right\rangle$. But, by definition of $W, C \in \mathfrak{P}^{* W}$ and thus also $C \in \mathfrak{P}^{*}\left\langle U_{1}, U_{2}\right\rangle$.

Proof of Proposition 3 The proof is formally the same of Proposition 10 in Bubboloni and Gori (2016b), simply taking into account (25).

Proof of Proposition 4 Let $C \in \mathfrak{P}^{* U}$ and $p \in \mathcal{P}$. Consider at first $(\varphi, \psi$, id $) \in U$. Since $C$ is $U$-symmetric then $C\left(p^{(\varphi, \psi, \text { id })}\right)=\psi C(p)$, that is, $C\left(p^{(\varphi, \psi, \text { id })}\right)=\{\psi q \in$ $\mathbf{L}(N): q \in C(p)\}$. Then,

$$
\begin{aligned}
C_{k}\left(p^{(\varphi, \psi, \mathrm{id})}\right) & =\left\{\{(\psi q)(r): 1 \leq r \leq k\} \in \mathbb{P}_{k}(N): q \in C(p)\right\} \\
& =\left\{\psi\{q(r): 1 \leq r \leq k\} \in \mathbb{P}_{k}(N): q \in C(p)\right\} \\
& =\psi\left\{\{q(r): 1 \leq r \leq k\} \in \mathbb{P}_{k}(N): q \in C(p)\right\}=\psi C_{k}(p),
\end{aligned}
$$

so that $C_{k}$ satisfies (8). In order to show that $C_{k}$ satisfies (9) too, assume by contradiction that $\left|C_{k}(p)\right|=1$ and that there exists $\left(\varphi, \psi, \rho_{0}\right) \in G$ such that $C_{k}\left(p^{\left(\varphi, \psi, \rho_{0}\right)}\right)=$ $\psi C_{k}(p)$. Then, there are $k$ distinct elements $x_{1}, \ldots, x_{k}$ of $N$ such that $C_{k}(p)=$ $\left\{\left\{x_{1}, \ldots, x_{k}\right\}\right\}$ and

$$
C_{k}\left(p^{\left(\varphi, \psi, \rho_{0}\right)}\right)=\left\{\left\{\psi\left(x_{1}\right), \ldots, \psi\left(x_{k}\right)\right\}\right\} .
$$

In particular, for every $q \in C(p)$, we have $\{q(1), \ldots, q(k)\}=\left\{x_{1}, \ldots, x_{k}\right\}$. By the $U$-symmetry of $C$, we have $C\left(p^{\left(\varphi, \psi, \rho_{0}\right)}\right)=\psi C(p) \rho_{0}$, and thus,

$$
C_{k}\left(p^{\left(\varphi, \psi, \rho_{0}\right)}\right)=\left\{\left\{q^{\prime}(1), \ldots, q^{\prime}(k)\right\} \in \mathbb{P}_{k}(N): q^{\prime} \in \psi C(p) \rho_{0}\right\} .
$$

Now if $q^{\prime} \in \psi C(p) \rho_{0}$ there exists $q \in C(p)$ such that $q^{\prime}=\psi q \rho_{0}$ and therefore $q^{\prime}(1)=\psi q \rho_{0}(1)=\psi q(n)$. Since $n>k$ and $q$ is a bijection, we have that $q(n) \notin$ $\{q(1), \ldots, q(k)\}=\left\{x_{1}, \ldots, x_{k}\right\}$. Thus, since also $\psi$ is a bijection, we also have $q^{\prime}(1)=\psi q(n) \notin\left\{\psi\left(x_{1}\right), \ldots, \psi\left(x_{k}\right)\right\}$. It follows that no element of $C_{k}\left(p^{\left(\varphi, \psi, \rho_{0}\right)}\right)$ can coincide with $\left\{\psi\left(x_{1}\right), \ldots, \psi\left(x_{k}\right)\right\}$, against (27).

Proof of Proposition 5 We show that, for every $p \in \mathcal{P}$ and $(\varphi, \psi, \rho) \in U$, $C^{R}\left(p^{(\varphi, \psi, \rho)}\right)=\psi C^{R}(p) \rho$. Fix $p \in \mathcal{P}$ and $(\varphi, \psi, \rho) \in U$ and prove first that 
$\psi C^{R}(p) \rho \subseteq C^{R}\left(p^{(\varphi, \psi, \rho)}\right)$. Pick $q \in C^{R}(p)$ and show that $\psi q \rho \in C^{R}\left(p^{(\varphi, \psi, \rho)}\right)$. We have $q \supseteq R(p)$, which implies $\psi q \rho \supseteq \psi R(p) \rho$. Since $R$ is $U$-symmetric, we then have $\psi q \rho \supseteq R\left(p^{(\varphi, \psi, \rho)}\right)$ and therefore $\psi q \rho \in C^{R}\left(p^{(\varphi, \psi, \rho)}\right)$.

Let us prove now the other inclusion $C^{R}\left(p^{(\varphi, \psi, \rho)}\right) \subseteq \psi C^{R}(p) \rho$. Let $\bar{p}=p^{(\varphi, \psi, \rho)}$ and note that $p=\bar{p}^{\left(\varphi^{-1}, \psi^{-1}, \rho\right)}$. Observe that, since $U$ is a group and $(\varphi, \psi, \rho) \in U$ also its inverse $(\varphi, \psi, \rho)^{-1}=\left(\varphi^{-1}, \psi^{-1}, \rho\right) \in U$. Thus, by the inclusion just proved, we get $\psi^{-1} C^{R}(\bar{p}) \rho \subseteq C^{R}\left(\bar{p}^{\left(\varphi^{-1}, \psi^{-1}, \rho\right)}\right)$, that is, $\psi^{-1} C^{R}\left(p^{(\varphi, \psi, \rho)}\right) \rho \subseteq C^{R}(p)$ and we conclude applying $\psi$ on the left and $\rho$ on the right to both sides of that inclusion.

Proof of Proposition 6 Repeat word by word the proof of Proposition 2 writing $\mathfrak{M}$ instead of $\mathfrak{P}$ and $R$ instead of $C$.

\section{B Proof of Theorems 7, 9 and 10}

The proofs of Theorems 7, 9 and 10 are definitely technical and require some preliminary work. We underline that the results we are going to prove are more general as they provide a method to potentially build and count all the resolute refinements.

\section{B.1 The role of the orbit representatives}

Let $U \leq G$ and $p \in \mathcal{P}$. The set $p^{U}=\left\{p^{g} \in \mathcal{P}: g \in U\right\}$ is called the $U$-orbit of $p$ and $\operatorname{Stab}_{U}(p)=\left\{g \in U: p^{g}=p\right\} \leq U$ is called the stabilizer of $p$ in $U$. Recall that, for every $g \in U$, we have $\operatorname{Stab}_{U}\left(p^{g}\right)=\operatorname{Stab}_{U}(p)^{g}$. The set $\mathcal{P}^{U}=\left\{p^{U}: p \in \mathcal{P}\right\}$ of the $U$-orbits is a partition of $\mathcal{P}$. We use $\mathcal{P}^{U}$ as set of indexes and denote its elements with $j$. A vector $\left(p^{j}\right)_{j \in \mathcal{P}^{U}} \in \times_{j \in \mathcal{P}^{U}} \mathcal{P}$ is called a system of representatives of the $U$-orbits if, for every $j \in \mathcal{P}^{U}, p^{j} \in j$. The set of the systems of representatives of the $U$-orbits is denoted by $\mathfrak{S}(U)$. If $\left(p^{j}\right)_{j \in \mathcal{P} U} \in \mathfrak{S}(U)$, then, for every $p \in \mathcal{P}$, there exist $j \in \mathcal{P}^{U}$ and $(\varphi, \psi, \rho) \in U$ such that $p=p^{j(\varphi, \psi, \rho)}$. Note that if $p^{j_{1}\left(\varphi_{1}, \psi_{1}, \rho_{1}\right)}=p^{j_{2}\left(\varphi_{2}, \psi_{2}, \rho_{2}\right)}$ for some $j_{1}, j_{2} \in \mathcal{P}^{U}$ and some $\left(\varphi_{1}, \psi_{1}, \rho_{1}\right),\left(\varphi_{2}, \psi_{2}, \rho_{2}\right) \in U$, then $j_{1}=j_{2}$ and, by (26), $\left(\varphi_{2}^{-1} \varphi_{1}, \psi_{2}^{-1} \psi_{1}, \rho_{2}^{-1} \rho_{1}\right) \in \operatorname{Stab}_{U}\left(p^{j_{1}}\right)$.

In this section, we present some results explaining how a correspondence $C \in$ $\mathfrak{P}^{* U} \cup \mathfrak{P}^{U} \cup \mathfrak{C}_{k}^{U}$ is determined by the values it assumes on a system of representatives of the $U$-orbits in $\mathcal{P}$. To that purpose, the first step is to split $\mathcal{P}^{U}$ into two parts $\mathcal{P}_{1}^{U}$ and $\mathcal{P}_{2}^{U}$, where

$$
\begin{aligned}
& \mathcal{P}_{1}^{U}=\left\{j \in \mathcal{P}^{U}: \forall p \in j, \operatorname{Stab}_{U}(p) \leq S_{h} \times S_{n} \times\{\mathrm{id}\}\right\}, \\
& \mathcal{P}_{2}^{U}=\left\{j \in \mathcal{P}^{U}: \forall p \in j, \operatorname{Stab}_{U}(p) \not \leq S_{h} \times S_{n} \times\{\mathrm{id}\}\right\} .
\end{aligned}
$$

Note that those sets are well defined because $U \cap\left(S_{h} \times S_{n} \times\{\right.$ id $\left.\}\right)$ is normal in $U$.

Moreover, $\mathcal{P}_{1}^{U} \cup \mathcal{P}_{2}^{U}=\mathcal{P}^{U}$ and $\mathcal{P}_{1}^{U} \cap \mathcal{P}_{2}^{U}=\varnothing$. In particular, $\mathcal{P}_{1}^{U}$ and $\mathcal{P}_{2}^{U}$ cannot be both empty. Obviously, if $U \leq S_{h} \times S_{n} \times\{$ id $\}$, then $\mathcal{P}_{2}^{U}=\varnothing$ and $\mathcal{P}^{U}=\mathcal{P}_{1}^{U} \neq \varnothing$. 
We recall a part of Proposition 24 in Bubboloni and Gori (2016b) which is interesting for our scope $\mathrm{e}^{20}$ :

$\mathcal{P}_{2}^{U} \neq \varnothing$ if and only if there exists $\left(\varphi, \psi, \rho_{0}\right) \in U$ such that $\psi$ is a conjugate of $\rho_{0}$.

Proposition 23 Let $U \leq G$ and $C, C^{\prime} \in \mathfrak{P}^{* U}$. Assume that there exists $\left(p^{j}\right)_{j \in \mathcal{P}^{U}} \in$ $\mathfrak{S}(U)$ such that, for every $j \in \mathcal{P}^{U}, C\left(p^{j}\right)=C^{\prime}\left(p^{j}\right)$. Then, $C=C^{\prime}$.

Proof Let $p \in \mathcal{P}$ and show that $C(p)=C^{\prime}(p)$. We know there exist $j \in \mathcal{P}^{U}$ and $(\varphi, \psi, \rho) \in U$ such that $p=p^{j(\varphi, \psi, \rho)}$. Then, $C(p)=C\left(p^{j(\varphi, \psi, \rho)}\right)=\psi C\left(p^{j}\right) \rho=$ $\psi C^{\prime}\left(p^{j}\right) \rho=C^{\prime}\left(p^{j(\varphi, \psi, \rho)}\right)=C^{\prime}(p)$.

Proposition 24 Let $U \leq S_{h} \times S_{n} \times\{\mathrm{id}\}$ and $C, C^{\prime} \in \mathfrak{P}^{U}\left(C, C^{\prime} \in \mathfrak{C}_{k}^{U}\right)$. Assume that there exists $\left(p^{j}\right)_{j \in \mathcal{P}^{U}} \in \mathfrak{S}(U)$ such that $C\left(p^{j}\right)=C^{\prime}\left(p^{j}\right)$ for all $j \in \mathcal{P}^{U}$. Then, $C=C^{\prime}$.

The proof of the above result is the same as the one of Proposition 12 in Bubboloni and Gori (2016b) and so is omitted.

Proposition 25 Let $U \leq G$ such that $U \not \leq S_{h} \times S_{n} \times\{\mathrm{id}\}, C, C^{\prime} \in \mathfrak{P}^{U}\left(C, C^{\prime} \in \mathfrak{C}_{k}^{U}\right)$. Assume that there exist $\left(p^{j}\right)_{j \in \mathcal{P}^{U}} \in \mathfrak{S}(U)$ and $\left(\varphi_{*}, \psi_{*}, \rho_{0}\right) \in U$ such that $C\left(p^{j}\right)=$ $C^{\prime}\left(p^{j}\right)$ for all $j \in \mathcal{P}^{U}$ and $C\left(p^{j\left(\varphi_{*}, \psi_{*}, \rho_{0}\right)}\right)=C^{\prime}\left(p^{j\left(\varphi_{*}, \psi_{*}, \rho_{0}\right)}\right)$ for all $j \in \mathcal{P}_{1}^{U}$. Then, $C=C^{\prime}$.

The proof of the above result is formally the same as the one of Proposition 13 in Bubboloni and Gori (2016b) and so is omitted.

\section{B.2 Resolute SPCs and $\boldsymbol{k}$-SCCs}

We have defined $C \in \mathfrak{P}\left(C \in \mathfrak{C}_{k}\right)$ resolute if, for every $p \in \mathcal{P},|C(p)|=1$. We now denote by $\mathfrak{F}\left(\mathfrak{F}_{k}\right)$ the set of resolute SPC $(k-\mathrm{SCC})$. Obviously, we have $\mathfrak{F} \subseteq \mathfrak{P}$ and $\mathfrak{F}_{k} \subseteq \mathfrak{C}_{k}$ and, for every $U \leq G$, we can consider the following sets

$$
\mathfrak{F}^{* U}=\mathfrak{F} \cap \mathfrak{P}^{* U}, \quad \mathfrak{F}^{U}=\mathfrak{F} \cap \mathfrak{P}^{U}, \quad \mathfrak{F}_{k}^{U}=\mathfrak{F}_{k} \cap \mathfrak{C}_{k}^{U}
$$

They describe, respectively, the set of the $U$-symmetric resolute SPC, the set of the $U$-consistent resolute SPC, the set of the $U$-consistent resolute $k$-SCC.

Given $C \in \mathfrak{F}$, for every $p \in \mathcal{P}$, there exists a unique $q \in \mathbf{L}(N)$ such that $C(p)=$ $\{q\}$. Thus, $C$ can be naturally identified with the social preference function (SPF) $f$ from $\mathcal{P}$ to $\mathbf{L}(N)$ defined, for every $p \in \mathcal{P}$, by $f(p)=q$. Similarly, given $C \in \mathfrak{F}_{k}$, for every $p \in \mathcal{P}$, there exists a unique $W \in \mathbb{P}_{k}(N)$ such that $C(p)=\{W\}$. Thus, $C$ can be naturally identified with the $k$-multiwinner social choice function ( $k$-SCF) $f$ from $\mathcal{P}$ to $\mathbb{P}_{k}(N)$ defined, for every $p \in \mathcal{P}$, by $f(p)=W$. We will freely adopt those identifications and the language of functions in what follows. For that reason,

20 Further details can be found in Section 5.2 of Bubboloni and Gori (2016b). 
we will refer to $\mathfrak{F}^{* U}$ also as the set of the $U$-symmetric SPFs; to $\mathfrak{F}^{U}$ also as the set of the $U$-consistent SPFs; to $\mathfrak{F}_{k}^{U}$ also as the set of the $U$-consistent $k$-SCFs.

Let $C \in \mathfrak{P}\left(C \in \mathfrak{C}_{k}\right)$. Denote the refinements of $C$ by $\mathfrak{P}_{C}\left(\mathfrak{C}_{k, C}\right)$. Then, the resolute refinements of $C$ are the functions in the set $\mathfrak{F}_{C}\left(\mathfrak{F}_{k, C}\right)$ defined by $\mathfrak{F}_{C}=\mathfrak{F} \cap \mathfrak{P}_{C}$ $\left(\mathfrak{F}_{k, C}=\mathfrak{F}_{k} \cap \mathfrak{C}_{k, C}\right)$.

Let now $U \leq G$. Given $C \in \mathfrak{P}$, we consider the following sets

$$
\mathfrak{F}_{C}^{* U}=\mathfrak{F}^{* U} \cap \mathfrak{F}_{C}, \quad \mathfrak{F}_{C}^{U}=\mathfrak{F}^{U} \cap \mathfrak{F}_{C} .
$$

They describe, respectively, the set of the $U$-symmetric SPFs which are refinements of $C$ and the set of the $U$-consistent SPFs which are refinements of $C$. Given $C \in \mathfrak{C}_{k}$, we finally consider the set

$$
\mathfrak{F}_{k, C}^{U}=\mathfrak{F}_{k}^{U} \cap \mathfrak{F}_{k, C}
$$

describing the set of $U$-consistent $k$-SCFs which are refinements of $C$.

\section{B.3 Proof of Theorem 7}

In order to approach the proof of Theorem 7, we need some preliminary facts. Let $U \leq G$ and $S \in \mathfrak{P}$ be defined, for every $p \in \mathcal{P}$, by

$$
S(p)=\left\{q \in \mathbf{L}(N): \forall(\varphi, \psi, \rho) \in \operatorname{Stab}_{U}(p), \psi q \rho=q\right\} .
$$

21

Proposition 26 Let $U \leq G$. Then, the following facts hold:

(i) $S$ is decisive if and only if $U$ is regular.

(ii) $S \in \mathfrak{P}^{* U}$.

(iii) If $f \in \mathfrak{F}^{* U}$, then $f \in \mathfrak{F}_{S}$.

Proof (i) Let $U$ be regular. Proving (11), in Bubboloni and Gori (2015), the authors showed that

$$
S(p)= \begin{cases}\mathbf{L}(N) & \text { if } \operatorname{Stab}_{U}(p) \leq S_{h} \times\{\mathrm{id}\} \times\{\mathrm{id}\} \\ u C_{S_{n}}\left(\rho_{0}\right) & \text { if } \operatorname{Stab}_{U}(p) \not \leq S_{h} \times\{\mathrm{id}\} \times\{\mathrm{id}\},\end{cases}
$$

where $C_{S_{n}}\left(\rho_{0}\right)$ denotes the centralizer of $\rho_{0}$ in $S_{n}$ and $u \in S_{n}$ is such that $\psi_{*}=u \rho_{0} u^{-1}$, with $\psi_{*} \in S_{n}$ the permutation appearing in the definition (10) of regularity. In particular,

$$
|S(p)|= \begin{cases}n ! & \text { if } \operatorname{Stab}_{U}(p) \leq S_{h} \times\{\text { id }\} \times\{\text { id }\} \\ 2^{\left\lfloor\frac{n}{2}\right\rfloor}\left\lfloor\frac{n}{2}\right\rfloor ! & \text { if } \operatorname{Stab}_{U}(p) \not S_{h} \times\{\text { id }\} \times\{\text { id }\} .\end{cases}
$$

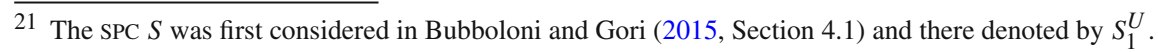


Thus, the fact that $S$ is decisive immediately follows from (29).

Assume now that $S$ is decisive. We need to show that, for every $p \in \mathcal{P}$, $(\varphi, \psi, \mathrm{id}) \in \operatorname{Stab}_{U}(p)$ implies $\psi=\mathrm{id}$, and $\left(\varphi, \psi, \rho_{0}\right) \in \operatorname{Stab}_{U}(p)$ implies $\psi=\psi_{*}$ for a suitable unique conjugate $\psi_{*}$ of $\rho_{0}$. Let $p \in \mathcal{P}$ and pick $q_{0} \in S(p)$. If $(\varphi, \psi$, id $) \in \operatorname{Stab}_{U}(p)$, then we have $\psi q_{0}=q_{0}$, and thus, by cancellation, $\psi=\mathrm{id}$. If $\left(\varphi, \psi, \rho_{0}\right) \in \operatorname{Stab}_{U}(p)$, then we have $\psi q_{0} \rho_{0}=q_{0}$, which implies $\psi=q_{0} \rho_{0} q_{0}^{-1}$. Thus, $\psi_{*}=q_{0} \rho_{0} q_{0}^{-1}$ works.

(ii) In order to show that $S$ is $U$-symmetric, we pick $\left(\varphi_{1}, \psi_{1}, \rho_{1}\right) \in U$ and see that $S\left(p^{\left(\varphi_{1}, \psi_{1}, \rho_{1}\right)}\right)=\psi_{1} S(p) \rho_{1}$. Recall that $\operatorname{Stab}_{U}\left(p^{\left(\varphi_{1}, \psi_{1}, \rho_{1}\right)}\right)=\operatorname{Stab}_{U}(p)^{\left(\varphi_{1}, \psi_{1}, \rho_{1}\right)}$. Thus, $q \in S\left(p^{\left(\varphi_{1}, \psi_{1}, \rho_{1}\right)}\right)$ if and only if, for every $(\varphi, \psi, \rho) \in \operatorname{Stab}_{U}(p)$, $\psi_{1} \psi \psi_{1}^{-1} q \rho_{1} \rho \rho_{1}^{-1}=q$, which is equivalent to $\psi\left(\psi_{1}^{-1} q \rho_{1}\right) \rho=\psi_{1}^{-1} q \rho_{1}$, that is, to $\psi_{1}^{-1} q \rho_{1} \in S(p)$ and thus to $q \in \psi_{1} S(p) \rho_{1}$.

(iii) This is just Lemma 4 in Bubboloni and Gori (2015).

By the above proposition, any $U$-symmetric SPF maps the profile $p$ into an element of $S(p)$. The next result shows that, conversely, one can construct $f \in \mathfrak{F}^{* U}$ just fixing a system $\left(p^{j}\right)_{j \in \mathcal{P}^{U}}$ of representatives and assigning within $S\left(p^{j}\right)$ the value to be assumed on each $p^{j}$. Its proof is formally equal to Proposition 5 in Bubboloni and Gori (2015).

Proposition 27 Let $U \leq G$ be regular and $\left(p^{j}\right)_{j \in \mathcal{P}^{U}} \in \mathfrak{S}(U)$. For every $j \in \mathcal{P}^{U}$, let $q_{j} \in S\left(p^{j}\right)$. Then there exists a unique $f \in \mathfrak{F}^{* U}$ such that, for every $j \in \mathcal{P}^{U}$, $f\left(p^{j}\right)=q_{j}$.

Proof Given $p \in \mathcal{P}$, there exist a unique $j \in \mathcal{P}^{U}$ such that $p=p^{j(\varphi, \psi, \rho)}$ for some $(\varphi, \psi, \rho) \in U$. We claim that, if for some $j \in \mathcal{P}^{U}$ there exist $\left(\varphi_{1}, \psi_{1}, \rho_{1}\right),\left(\varphi_{2}, \psi_{2}, \rho_{2}\right) \in U$ such that $p^{j\left(\varphi_{1}, \psi_{1}, \rho_{1}\right)}=p^{j\left(\varphi_{2}, \psi_{2}, \rho_{2}\right)}$, then $\psi_{1} q_{j} \rho_{1}=$ $\psi_{2} q_{j} \rho_{2}$. Indeed, by (26), we have that $p^{j\left(\varphi_{1}, \psi_{1}, \rho_{1}\right)}=p^{j\left(\varphi_{2}, \psi_{2}, \rho_{2}\right)}$ implies $\left(\varphi_{2}^{-1} \varphi_{1}, \psi_{2}^{-1} \psi_{1}, \rho_{2}^{-1} \rho_{1}\right) \in \operatorname{Stab}_{U}\left(p^{j}\right)$. Since $q_{j} \in S\left(p^{j}\right)$ and $\Omega$ is abelian, we have that $q_{j}=\psi_{2}^{-1} \psi_{1} q_{j} \rho_{2}^{-1} \rho_{1}=\psi_{2}^{-1} \psi_{1} q_{j} \rho_{1} \rho_{2}^{-1}$, and thus $\psi_{1} q_{j} \rho_{1}=\psi_{2} q_{j} \rho_{2}$.

As a consequence, the resolute SPC $f$ defined, for every $p \in \mathcal{P}$, by $f(p)=\psi q_{j} \rho$, where $j \in \mathcal{P}^{U}$ and $(\varphi, \psi, \rho) \in U$ are such that $p=p^{j(\varphi, \psi, \rho)}$, is consistent. Moreover, for every $j \in \mathcal{P}^{U}, f\left(p^{j}\right)=q_{j}$. Let us prove that $f \in \mathfrak{P}^{* U}$. Consider $p \in \mathcal{P}$ and $(\varphi, \psi, \rho) \in U$. Let $p=p^{j\left(\varphi_{1}, \psi_{1}, \rho_{1}\right)}$, for some $j \in \mathcal{P}^{U}$ and $\left(\varphi_{1}, \psi_{1}, \rho_{1}\right) \in U$. By the definition of $f$ and by (26) and using again the fact that $\Omega$ is abelian, we have

$$
\begin{aligned}
f\left(p^{(\varphi, \psi, \rho)}\right) & =f\left(\left(p^{j\left(\varphi_{1}, \psi_{1}, \rho_{1}\right)}\right)^{(\varphi, \psi, \rho)}\right)=f\left(p^{j\left(\varphi \varphi_{1}, \psi \psi_{1}, \rho \rho_{1}\right)}\right)=\left(\psi \psi_{1}\right) q_{j}\left(\rho \rho_{1}\right) \\
& =\left(\psi \psi_{1}\right) q_{j}\left(\rho_{1} \rho\right)=\psi\left(\psi_{1} q_{j} \rho_{1}\right) \rho=\psi f\left(p^{j\left(\varphi_{1}, \psi_{1}, \rho_{1}\right)}\right) \rho=\psi f(p) \rho .
\end{aligned}
$$

In order to prove the uniqueness of $f$, it suffices to note that if $f^{\prime} \in \mathfrak{F}^{* U}$ is such that, for every $j \in \mathcal{P}^{U}, f^{\prime}\left(p^{j}\right)=q_{j}$, then we have $f=f^{\prime}$ by Proposition 23 .

Given now $\left(p^{j}\right)_{j \in \mathcal{P}^{U}} \in \mathfrak{S}(U)$, let

$$
\Phi: \times_{j \in \mathcal{P}^{U}} S\left(p^{j}\right) \rightarrow \mathfrak{F}^{* U}
$$


be the function which associates with every $\left(q_{j}\right)_{j \in \mathcal{P}^{U}} \in \times_{j \in \mathcal{P}^{U}} S\left(p^{j}\right)$ the unique $f \in \mathfrak{F}^{* U}$ defined in Proposition 27. Of course, $\Phi$ depends on $\left(p^{j}\right)_{j \in \mathcal{P}^{U}}$ but we do not emphasize that dependence in the notation. Note that $\Phi$ is injective.

Theorem 28 Let $U \leq G$ be regular, $\left(p^{j}\right)_{j \in \mathcal{P}^{U}} \in \mathfrak{S}(U)$ and $C \in \mathfrak{P}^{*}$. Then

$$
\mathfrak{F}_{C}^{* U}=\Phi\left(\times_{j \in \mathcal{P} U} S\left(p^{j}\right) \cap C\left(p^{j}\right)\right)
$$

and

$$
\left|\mathfrak{F}_{C}^{* U}\right|=\prod_{j \in \mathcal{P}^{U}}\left|S\left(p^{j}\right) \cap C\left(p^{j}\right)\right| .
$$

Proof We first prove that $\Phi\left(\times_{j \in \mathcal{P}^{U}} S\left(p^{j}\right) \cap C\left(p^{j}\right)\right) \subseteq \mathfrak{F}_{C}^{* U}$. Let $\left(q_{j}\right)_{j \in \mathcal{P}^{U}} \in$ $\times_{j \in \mathcal{P}^{U}} S\left(p^{j}\right) \cap C\left(p^{j}\right)$ and $f=\Phi\left(\left(q_{j}\right)_{j \in \mathcal{P}^{U}}\right)$. We show that $f \in \mathfrak{F}_{C}^{* U}$. We know that $f \in \mathfrak{F}^{* U}$, so that have are left with showing that $f \in \mathfrak{F}_{C}$. Given $p \in \mathcal{P}$, there exist $j \in \mathcal{P}^{U}$ and $(\varphi, \psi, \rho) \in U$ such that $p=p^{j(\varphi, \psi, \rho)}$. As we know that $q_{j}=f\left(p^{j}\right) \in C\left(p^{j}\right)$, by $U$-symmetry of $f$ and $C$, we have

$$
f(p)=f\left(p^{j(\varphi, \psi, \rho)}\right)=\psi q_{j} \rho \in \psi C\left(p^{j}\right) \rho=C\left(p^{j(\varphi, \psi, \rho)}\right)=C(p)
$$

as desired.

Let us next prove that $\mathfrak{F}_{C}^{* U} \subseteq \Phi\left(\times_{j \in \mathcal{P}^{U}} S\left(p^{j}\right) \cap C\left(p^{j}\right)\right)$. Consider then $f \in$ $\mathfrak{F}_{C}^{* U}$ and note that, by Proposition 26(iii), for every $j \in \mathcal{P}^{U}, f\left(p^{j}\right) \in S\left(p^{j}\right) \cap$ $C\left(p^{j}\right)$. Then $\left(f\left(p^{j}\right)\right)_{j \in \mathcal{P}^{U}} \in \times_{j \in \mathcal{P}^{U}} S\left(p^{j}\right) \cap C\left(p^{j}\right)$. Thus, the function $f$ and the function $\Phi\left(\left(f\left(p^{j}\right)\right)_{j \in \mathcal{P}^{U}}\right)$ are $U$-symmetric functions which coincide on a system of representatives. Hence, by Proposition 23, we obtain $f=\Phi\left(\left(f\left(p^{j}\right)\right)_{j \in \mathcal{P}^{U}}\right)$.

The last part of the statement is an immediate consequence of the fact that $\Phi$ is injective.

In order to write down the proof of Theorem 7, we need a final technical lemma.

Lemma 29 Let $R \in \mathfrak{M}^{* U}$ and $p \in \mathcal{P}$. Then, for every $x, y \in N$ and $\left(\varphi, \psi, \rho_{0}\right) \in$ $\operatorname{Stab}_{U}(p),(x, y) \in R(p)$ if and only if $(\psi(y), \psi(x)) \in R(p)$.

Proof Let $x, y \in N$ and $\left(\varphi, \psi, \rho_{0}\right) \in \operatorname{Stab}_{U}(p)$. Then, by the $U$-symmetry of $R$, we have $R(p)=R\left(p^{\left(\varphi, \psi, \rho_{0}\right)}\right)=\psi R(p) \rho_{0}$. On the other hand, $x \succeq_{R(p)} y$ is equivalent to $\psi(y) \succeq_{\psi R(p) \rho_{0}} \psi(x)$ and thus to $\psi(y) \succeq_{R(p)} \psi(x)$.

Proof of Theorem 7 (i) $\Rightarrow$ (ii). The fact that $C \in \mathfrak{P}^{* U}$ admits a $U$-symmetric resolute refinement means that $\mathfrak{F}_{C}^{* U} \neq \varnothing$. Let then $f \in \mathfrak{F}_{C}^{* U}$ and define the social method $R: \mathcal{P} \rightarrow \mathbf{R}(N)$, by $R(p)=f(p) \backslash \Delta$ for all $p \in \mathcal{P}$, where $\Delta=\{(x, x): x \in N\}$. Note that $\Delta$ is a relation on $N$ and that for every $\psi \in S_{n}$ and $\rho \in \Omega$, we have $\psi \Delta \rho=\Delta$, accordingly to the definitions given in Sect. 2. We show that $R$ is irreflexive, acyclic, $U$-symmetric and that $C^{R}$ refines $C$. 
Let $p \in \mathcal{P} . R(p)$ is irreflexive by definition and surely acyclic since it refines the linear order $f(p)$. Let $(\varphi, \psi, \rho) \in U$. Then, by the $U$-symmetry of $f$, we have

$$
R\left(p^{(\varphi, \psi, \rho)}\right)=f\left(p^{(\varphi, \psi, \rho)}\right) \backslash \Delta=(\psi f(p) \rho) \backslash(\psi \Delta \rho)=\psi(f(p) \backslash \Delta) \rho=\psi R(p) \rho .
$$

Thus, $R \in \mathfrak{M}^{* U}$. Finally, observe that

$$
C^{R}(p)=\{q \in \mathbf{L}(N): R(p) \subseteq q\}=\{q \in \mathbf{L}(N): f(p) \backslash \Delta \subseteq q\}=\{f(p)\} \subseteq C(p)
$$

Thus $C^{R}$ refines $C$.

(ii) $\Rightarrow$ (iii) Let $R$ be an irreflexive, acyclic, $U$-symmetric social method such that $C^{R}$ refines $C$. Then, by Lemma 29, condition (a) in (iii) is fulfilled.

(iii) $\Rightarrow$ (i) The proof is a remake of Bubboloni and Gori (2015, Section 8), which is essentially obtained replacing the minimal majority relation $R^{\nu(p)}(p)$ there, with the present relation $R(p)$.

\section{B.4 Analysis of $\mathfrak{F}_{C}^{U}$ and $\mathfrak{F}_{k, C}^{U}$ when $U \leq S_{h} \times S_{n} \times\{$ id $\}$}

The next result, whose proof is similar to the one of Proposition 17 in Bubboloni and Gori (2016b), is very important.

Proposition 30 Let $U \leq S_{h} \times S_{n} \times\{\mathrm{id}\}$ be regular, $\left(p^{j}\right)_{j \in \mathcal{P}^{U}} \in \mathfrak{S}(U)$ and $C \in \mathfrak{P}^{U}$ $\left(C \in \mathfrak{C}_{k}^{U}\right)$. For every $j \in \mathcal{P}^{U}$, let $x_{j} \in C\left(p^{j}\right)$. Then there exists a unique $f \in \mathfrak{F}_{C}^{U}$ $\left(f \in \mathfrak{F}_{k, C}^{U}\right)$ such that, for every $j \in \mathcal{P}^{U}, f\left(p^{j}\right)=x_{j}$.

Proof Let $C \in \mathfrak{P}^{U}$. Consider $f \in \mathfrak{F}$ defined as follows. Given $p \in \mathcal{P}$, consider the unique $j \in \mathcal{P}^{U}$ such that $p \in j$ and the nonempty set $U_{p}=\{(\varphi, \psi$, id $) \in U$ : $\left.p=p^{j(\varphi, \psi, \text { id })}\right\}$. Pick $(\varphi, \psi$, id $) \in U_{p}$ and let $f(p)=\psi x_{j}$. We need to prove that the value of $f(p)$ does not depend on the particular element chosen in $U_{p}$. Indeed, let $\left(\varphi_{1}, \psi_{1}, \mathrm{id}\right),\left(\varphi_{2}, \psi_{2}, \mathrm{id}\right) \in U_{p}$ and note that $\left(\varphi_{2}^{-1} \varphi_{1}, \psi_{2}^{-1} \psi_{1}, \mathrm{id}\right) \in \operatorname{Stab}_{U}\left(p^{j}\right)$. Since $U$ is regular, that gives $\psi_{1}=\psi_{2}$ and then $\psi_{1} x_{j}=\psi_{2} x_{j}$.

We show that $f$ satisfies all the desired properties. Since $U \leq G$, we have (id, id, id) $\in U$ and thus the definition of $f$ immediately implies $f\left(p^{j}\right)=x_{j}$. Let us prove that $f \in \mathfrak{F}^{U}$. Consider $p \in \mathcal{P}$ and $(\varphi, \psi$, id $) \in U$ and show that $f\left(p^{(\varphi, \psi, \text { id })}\right)=$ $\psi f(p)$. Let $p=p^{j\left(\varphi_{1}, \psi_{1}, \text { id }\right)}$ for suitable $j \in \mathcal{P}^{U}$ and $\left(\varphi_{1}, \psi_{1}\right.$, id $) \in U$. Thus, $f(p)=\psi_{1} x_{j}$ and, by (26), $f\left(p^{(\varphi, \psi, \text { id })}\right)=f\left(p^{j\left(\varphi \varphi_{1}, \psi \psi_{1}, \text { id }\right)}\right)=\psi \psi_{1} x_{j}=\psi f(p)$.

Let us next prove that $f \in \mathfrak{F}_{C}$. Consider then $p \in \mathcal{P}$ and show that $f(p) \in C(p)$. Let $p=p^{j\left(\varphi_{1}, \psi_{1}, \text { id }\right)}$ for suitable $j \in \mathcal{P}^{U}$ and $\left(\varphi_{1}, \psi_{1}\right.$, id $) \in U$. Thus, $f(p)=\psi_{1} x_{j}$ and, since $C$ is $U$-consistent, $\psi_{1} x_{j} \in \psi_{1} C\left(p^{j}\right)=C\left(p^{j\left(\varphi_{1}, \psi_{1}, \text { id }\right)}\right)=C(p)$. Finally, in order to prove uniqueness, let $f^{\prime} \in \mathfrak{F}_{C}^{U}$ such that $f^{\prime}\left(p^{j}\right)=x_{j}$ for all $j \in \mathcal{P}^{U}$. Then $f^{\prime}$ and $f$ coincides on $\left(p^{j}\right)_{j \in \mathcal{P} U} \in \mathfrak{S}(U)$ and Proposition 24 applies giving $f^{\prime}=f$.

Let now $C \in \mathfrak{C}_{k}^{U}$. The proposition can be proved using the same argument. 
Let $U \leq S_{h} \times S_{n} \times\{$ id $\}$ be regular, $\left(p^{j}\right)_{j \in \mathcal{P}^{U}} \in \mathfrak{S}(U)$ and $C \in \mathfrak{P}^{U}\left(C \in \mathfrak{C}_{k}^{U}\right)$. Consider the function

$$
\Phi: \times_{j \in \mathcal{P}^{U}} C\left(p^{j}\right) \rightarrow \mathfrak{F}_{C}^{U}, \quad\left(\Phi: \times_{j \in \mathcal{P}^{U}} C\left(p^{j}\right) \rightarrow \mathfrak{F}_{k, C}^{U}\right)
$$

which associates with every $\left(x_{j}\right)_{j \in \mathcal{P}^{U}} \in \times_{j \in \mathcal{P}^{U}} C\left(p^{j}\right)$ the unique $f \in \mathfrak{F}_{C}^{U}\left(f \in \mathfrak{F}_{k, C}^{U}\right)$ defined in Proposition 30. Of course, $\Phi$ depends on $U,\left(p^{j}\right)_{j \in \mathcal{P} U}$ and $C$ but we do not emphasize that dependence in the notation. Note that we used the same letter $\Phi$ to treat both SPFs and $k$-SCFs. Note also that $\Phi$ is injective.

Theorem 31 Let $U \leq S_{h} \times S_{n} \times\{$ id $\}$ be regular, $\left(p^{j}\right)_{j \in \mathcal{P}^{U}} \in \mathfrak{S}(U)$ and $C \in \mathfrak{P}^{U}$ $\left(C \in \mathfrak{C}_{k}^{U}\right)$. Then

$$
\mathfrak{F}_{C}^{U}=\Phi\left(\times_{j \in \mathcal{P}^{U}} C\left(p^{j}\right)\right) \quad\left(\mathfrak{F}_{k, C}^{U}=\Phi\left(\times_{j \in \mathcal{P}^{U}} C\left(p^{j}\right)\right)\right)
$$

and

$$
\left|\mathfrak{F}_{C}^{U}\right|=\prod_{j \in \mathcal{P}^{U}}\left|C\left(p^{j}\right)\right| \quad\left(\left|\mathfrak{F}_{k, C}^{U}\right|=\prod_{j \in \mathcal{P}^{U}}\left|C\left(p^{j}\right)\right|\right) .
$$

In particular, if $C$ is decisive, then $\mathfrak{F}_{C}^{U} \neq \varnothing\left(\mathfrak{F}_{k, C}^{U} \neq \varnothing\right)$.

The proof of the above result is similar to the one of Theorem 18 in Bubboloni and Gori (2016b) and thus omitted.

\section{B.5 Analysis of $\mathfrak{F}_{C}^{U}$ and $\mathfrak{F}_{k, C}^{U}$ when $U \not \leq S_{h} \times S_{n} \times\{$ id $\}$}

Let $U \leq G$ be regular such that $U \not \leq S_{h} \times S_{n} \times\{\mathrm{id}\}, C \in \mathfrak{P}^{U}\left(C \in \mathfrak{C}_{k}^{U}\right)$. Recall that if $p \in \overline{\mathcal{P}}$ and $y \in C(p)$, then $y \in \mathbf{L}(N)\left(y \in \mathbb{P}_{k}(N)\right)$. Moreover, given $\psi \in S_{n}$, the meaning of the writing $\psi y$ is carefully explained in Sect. 2.

Fix $\left(p^{j}\right)_{j \in \mathcal{P}^{U}} \in \mathfrak{S}(U)$ and $\left(\varphi_{*}, \psi_{*}, \rho_{0}\right) \in U$. Define, for every $j \in \mathcal{P}_{1}^{U}$, the set

$$
A_{C}^{1}\left(p^{j}\right)=\left\{(y, z) \in C\left(p^{j}\right) \times C\left(p^{j\left(\varphi_{*}, \psi_{*}, \rho_{0}\right)}\right): z \neq \psi_{*} y\right\},
$$

and, for every $j \in \mathcal{P}_{2}^{U}$, the set

$$
A_{C}^{2}\left(p^{j}\right)=\left\{x \in C\left(p^{j}\right): \psi_{j} x \neq x\right\}
$$

where $\psi_{j}$ is the unique element in $S_{n}$ such that

$$
\operatorname{Stab}_{U}\left(p^{j}\right) \subseteq\left(S_{h} \times\{\mathrm{id}\} \times\{\mathrm{id}\}\right) \cup\left(S_{h} \times\left\{\psi_{j}\right\} \times\left\{\rho_{0}\right\}\right)
$$

Note that the uniqueness of $\psi_{j}$ is guaranteed by Lemma 16 (ii) in Bubboloni and Gori (2016b). 
Next if $\mathcal{P}_{1}^{U} \neq \varnothing$, then define

$$
A_{C}^{1}=\times_{j \in \mathcal{P}_{1}^{U}} A_{C}^{1}\left(p^{j}\right)
$$

and if $\mathcal{P}_{2}^{U} \neq \varnothing$, then define

$$
A_{C}^{2}=\times_{j \in \mathcal{P}_{2}^{U}} A_{C}^{2}\left(p^{j}\right)
$$

Recall that $\mathcal{P}_{1}^{U}$ and $\mathcal{P}_{2}^{U}$ cannot be both empty. Thus, at least one of the above sets is always defined. Of course, $A_{C}^{1}$ and $A_{C}^{2}$ depend also on $U,\left(p^{j}\right)_{j \in \mathcal{P}^{U}}$ and $\left(\varphi_{*}, \psi_{*}, \rho_{0}\right)$ but we do not emphasize that dependence in the notation.

The proof of the next important result is similar to Proposition 19 in Bubboloni and Gori (2016b).

Proposition 32 Let $U \leq G$ be regular such that $U \not \leq S_{h} \times S_{n} \times\{\mathrm{id}\},\left(p^{j}\right)_{j \in \mathcal{P} U} \in$ $\mathfrak{S}(U),\left(\varphi_{*}, \psi_{*}, \rho_{0}\right) \in U$ and $C \in \mathfrak{P}^{U}\left(C \in \mathfrak{C}_{k}^{U}\right)$. For every $j \in \mathcal{P}_{1}^{U}$, let $\left(y_{j}, z_{j}\right) \in$ $A_{C}^{1}\left(p^{j}\right)$ and, for every $j \in \mathcal{P}_{2}^{U}$, let $x_{j} \in A_{C}^{2}\left(p^{j}\right)$. Then, there exists a unique $f \in \mathfrak{F}_{C}^{U}$ $\left(f \in \mathfrak{F}_{k, C}^{U}\right)$ such that $f\left(p^{j}\right)=y_{j}$ and $f\left(p^{j\left(\varphi_{*}, \psi_{*}, \rho_{0}\right)}\right)=z_{j}$ for all $j \in \mathcal{P}_{1}^{U}$, and $f\left(p^{j}\right)=x_{j}$ for all $j \in \mathcal{P}_{2}^{U}$.

Proof Let $C \in \mathfrak{P}^{U}$. Given $j \in \mathcal{P}_{2}^{U}$, consider the set $K^{U}\left(p^{j}\right)=\left\{\sigma \in S_{n}: \psi_{j}=\right.$ $\left.\sigma \rho_{0} \sigma^{-1}\right\}$, where $\psi_{j}$ is defined in (30). Since $U$ is regular, $K^{U}\left(p^{j}\right)$ is nonempty so that we can choose an element $\sigma_{j}$ in $K^{U}\left(p^{j}\right)$. Note that, for every $j \in \mathcal{P}_{2}^{U}$ and $(\varphi, \psi, \rho) \in \operatorname{Stab}_{U}\left(p^{j}\right)$, we have $\psi=\sigma_{j} \rho \sigma_{j}^{-1}$.

Let us consider then $f \in \mathfrak{F}$ defined, for every $p \in \mathcal{P}$, as follows. Given $p \in \mathcal{P}$, consider the unique $j \in \mathcal{P}^{U}$ such that $p \in j$ and the nonempty set $U_{p}=\{(\varphi, \psi, \rho) \in$ $\left.U: p=p^{j(\varphi, \psi, \rho)}\right\}$. Pick $(\varphi, \psi, \rho) \in U_{p}$ and let

$$
f(p)= \begin{cases}\psi y_{j} & \text { if } j \in \mathcal{P}_{1}^{U} \text { and } \rho=\mathrm{id} \\ \psi \psi_{*}^{-1} z_{j} & \text { if } j \in \mathcal{P}_{1}^{U} \text { and } \rho=\rho_{0} \\ \psi \sigma_{j} \rho \sigma_{j}^{-1} x_{j} & \text { if } j \in \mathcal{P}_{2}^{U}\end{cases}
$$

We need to prove that the value of $f(p)$ does not depend on the particular element chosen in $U_{p}$ and that $f$ satisfies all the desired properties. That can be done following the same reasoning used in the proof of Proposition 19 in Bubboloni and Gori (2016b).

Let $U \leq G$ be regular such that $U \not \leq S_{h} \times S_{n} \times\{\mathrm{id}\},\left(p^{j}\right)_{j \in \mathcal{P} U} \in \mathfrak{S}(U)$, $\left(\varphi_{*}, \psi_{*}, \rho_{0}\right) \in U$ and $C \in \mathfrak{P}^{U}\left(C \in \mathfrak{C}_{k}^{U}\right)$.

- If $\mathcal{P}_{2}^{U}=\varnothing$, then let $\Psi_{1}: A_{C}^{1} \rightarrow \mathfrak{F}_{C}^{U}\left(\Psi_{1}: A_{C}^{1} \rightarrow \mathfrak{F}_{k, C}^{U}\right)$ be the function which associates with every $\left(y_{j}, z_{j}\right)_{j \in \mathcal{P}_{1}^{U}} \in A_{C}^{1}$, the unique $f \in \mathfrak{F}_{C}^{U}\left(f \in \mathfrak{F}_{k, C}^{U}\right)$ defined in Proposition 32. 
- If $\mathcal{P}_{1}^{U}=\varnothing$, then let $\Psi_{2}: A_{C}^{2} \rightarrow \mathfrak{F}_{C}^{U}\left(\Psi_{2}: A_{C}^{2} \rightarrow \mathfrak{F}_{k, C}^{U}\right)$ be the function which associates with every $\left(x_{j}\right)_{j \in \mathcal{P}_{2}^{U}} \in A_{C}^{2}$, the unique $f \in \mathfrak{F}_{C}^{U}\left(f \in \mathfrak{F}_{k, C}^{U}\right)$ defined in Proposition 32.

- If $\mathcal{P}_{1}^{U} \neq \varnothing$ and $\mathcal{P}_{2}^{U} \neq \varnothing$, then let $\Psi_{3}: A_{C}^{1} \times A_{C}^{2} \rightarrow \mathfrak{F}_{C}^{U}\left(\Psi_{3}: A_{C}^{1} \times A_{C}^{2} \rightarrow \mathfrak{F}_{k, C}^{U}\right)$ be the function which associates with every $\left(\left(y_{j}, z_{j}\right)_{j \in \mathcal{P}_{1}^{U}},\left(x_{j}\right)_{j \in \mathcal{P}_{2}^{U}}\right) \in A_{C}^{1} \times A_{C}^{2}$, the unique $f \in \mathfrak{F}_{C}^{U}\left(f \in \mathfrak{F}_{k, C}^{U}\right)$ defined in Proposition 32.

Of course, $\Psi_{1}, \Psi_{2}$ and $\Psi_{3}$ depend on $U,\left(p^{j}\right)_{j \in \mathcal{P}^{U}},\left(\varphi_{*}, \psi_{*}, \rho_{0}\right)$ and $C$ but we do not emphasize that dependence in the notation. Note also that $\Psi_{1}, \Psi_{2}$ and $\Psi_{3}$ are injective.

Theorem 33 Let $U \leq G$ be regular such that $U \not \leq S_{h} \times S_{n} \times\{\mathrm{id}\},\left(p^{j}\right)_{j \in \mathcal{P} U} \in \mathfrak{S}(U)$, $\left(\varphi_{*}, \psi_{*}, \rho_{0}\right) \in U$ and $C \in \mathfrak{P}^{U}\left(C \in \mathfrak{C}_{k}^{U}\right)$. Then

$$
\mathfrak{F}_{C}^{U}\left(\mathfrak{F}_{k, C}^{U}\right)= \begin{cases}\Psi_{1}\left(A_{C}^{1}\right) & \text { if } \mathcal{P}_{2}^{U}=\varnothing \\ \Psi_{2}\left(A_{C}^{2}\right) & \text { if } \mathcal{P}_{1}^{U}=\varnothing \\ \Psi_{3}\left(A_{C}^{1} \times A_{C}^{2}\right) & \text { if } \mathcal{P}_{1}^{U} \neq \varnothing \text { and } \mathcal{P}_{2}^{U} \neq \varnothing\end{cases}
$$

and

$$
\left|\mathfrak{F}_{C}^{U}\right|\left(\left|\mathfrak{F}_{k, C}^{U}\right|\right)= \begin{cases}\left|A_{C}^{1}\right| & \text { if } \mathcal{P}_{2}^{U}=\varnothing \\ \left|A_{C}^{2}\right| & \text { if } \mathcal{P}_{1}^{U}=\varnothing \\ \left|A_{C}^{1}\right| \cdot\left|A_{C}^{2}\right| & \text { if } \mathcal{P}_{1}^{U} \neq \varnothing \text { and } \mathcal{P}_{2}^{U} \neq \varnothing\end{cases}
$$

Moreover, if $C$ is decisive, then we have that:

- for every $j \in \mathcal{P}_{1}^{U}, A_{C}^{1}\left(p^{j}\right) \neq \varnothing$,

- $\mathfrak{F}_{C}^{U} \neq \varnothing\left(\mathfrak{F}_{k, C}^{U} \neq \varnothing\right)$ if and only if, for every $j \in \mathcal{P}_{2}^{U}, A_{C}^{2}\left(p^{j}\right) \neq \varnothing$.

Proof Let $C \in \mathfrak{P}^{U}$. Assume first that $\mathcal{P}_{1}^{U}$ and $\mathcal{P}_{2}^{U}$ are both nonempty. Consider $f \in \mathfrak{F}_{C}^{U}$ and note that

$$
\left(\left(f\left(p^{j}\right), f\left(p^{j\left(\varphi_{*}, \psi_{*}, \rho_{0}\right)}\right)\right)_{j \in \mathcal{P}_{1}^{U}},\left(f\left(p^{j}\right)\right)_{j \in \mathcal{P}_{2}^{U}}\right) \in A_{C}^{1} \times A_{C}^{2},
$$

and

$$
\Psi_{3}\left(\left(f\left(p^{j}\right), f\left(p^{j\left(\varphi_{*}, \psi_{*}, \rho_{0}\right)}\right)\right)_{j \in \mathcal{P}_{1}^{U}},\left(f\left(p^{j}\right)\right)_{j \in \mathcal{P}_{2}^{U}}\right)=f .
$$

Thus, $\Psi_{3}$ is bijective and we have $\left|\mathfrak{F}_{C}^{U}\right|=\left|A_{C}^{1} \times A_{C}^{2}\right|=\left|A_{C}^{1}\right| \cdot\left|A_{C}^{2}\right|$. The case $\mathcal{P}_{1}^{U}=\varnothing$ and the case $\mathcal{P}_{2}^{U}=\varnothing$ are similar and then omitted.

Assume now that $C$ is decisive. Assume, by contradiction, that there exists $j \in \mathcal{P}_{1}^{U}$ such that $A_{C}^{1}\left(p^{j}\right)=\varnothing$. Thus, for every $y \in C\left(p^{j}\right)$ and $z \in C\left(p^{j\left(\varphi_{*}, \psi_{*}, \rho_{0}\right)}\right)$ we have $z=\psi_{*} y$. Using decisiveness, fix $z \in C\left(p^{j\left(\varphi_{*}, \psi_{*}, \rho_{0}\right)}\right)$. If $y_{1}, y_{2} \in C\left(p^{j}\right)$, we then have $z=\psi_{*} y_{1}=\psi_{*} y_{2}$ so that $y_{1}=y_{2}$. It follows that $\left|C\left(p^{j}\right)\right|=1=\left|C\left(p^{j\left(\varphi_{*}, \psi_{*}, \rho_{0}\right)}\right)\right|$ and that $C\left(p^{j\left(\varphi_{*}, \psi_{*}, \rho_{0}\right)}\right)=\psi_{*} C\left(p^{j}\right)$, against $U$-consistency. The last part of the theorem is trivial. 
Let now consider $C \in \mathfrak{C}_{k}^{U}$. The theorem can be proved using formally the same argument.

\section{B.6 Proof of Theorem 9}

Proof of Theorem 9 Let first $U \leq S_{h} \times S_{n} \times\{$ id $\}$. Then, by Theorem 31, we have that $\mathfrak{F}_{C}^{U} \neq \varnothing$. Let next $U \nless \leq S_{h} \times S_{n} \times\{\mathrm{id}\}$. In order to show that also in this case we have $\mathfrak{F}_{C}^{U} \neq \varnothing$, by Theorem 33, we need to prove that, for every $j \in \mathcal{P}_{2}^{U}, A_{C}^{2}\left(p^{j}\right) \neq \varnothing$. Assume, by contradiction, that there exists $j \in \mathcal{P}_{2}^{U}$ such that $A_{C}^{2}\left(p^{j}\right)=\varnothing$ and consider $\psi_{j} \in S_{n}$ as defined in (30). Then, for every $x \in C\left(p^{j}\right)$, we have $\psi_{j} x=x$. Recall that $\psi_{j}$ is a conjugate of $\rho_{0}$, and thus, in particular, $\psi_{j} \neq \mathrm{id}$. Since $C$ is decisive, we can pick $x \in C\left(p^{j}\right)$. Thus, $x \in S_{n}$ and, using the cancellation law in $S_{n}$, we get the contradiction $\psi_{j}=\mathrm{id}$.

\section{B.7 Proof of Theorem 10}

Theorem 33 says that to guarantee the existence of a $U$-consistent resolute refinement for some $C \in \mathfrak{C}_{k}^{U} \cup \mathfrak{P}^{U}$, we need to satisfy the condition $A_{C}^{2}\left(p^{j}\right) \neq \varnothing$ for every $j \in \mathcal{P}_{2}^{U}$, where $\left(p^{j}\right)_{j \in \mathcal{P} U} \in \mathfrak{S}(U)$. We have seen in the proof of Theorem 9 , that that condition always holds if $C \in \mathfrak{P}^{U}$. On the other hand, there is no reason for having it satisfied when $C \in \mathfrak{C}_{k}^{U}$. Indeed, in that context, $A_{C}^{2}\left(p^{j}\right)=\varnothing$ for some $j \in \mathcal{P}_{2}^{U}$ means that for $\psi_{j} \in S_{n}$ defined by (30) we have

$$
\text { for every } x \in C\left(p^{j}\right), \quad \psi_{j} x=x \text {. }
$$

In other words, $\psi_{j}$ fixes all the $k$-subsets of $N$ appearing in $C\left(p^{j}\right)$. Thus, the elements of $C\left(p^{j}\right)$ need to be union of $\psi_{j}$-orbits and this does not constitute, in principle, a contradictory fact.

The situation is then more variegated with respect to the case of the SPCs and, in order to manage it, we need some preliminary work. The next lemma is an easy but very useful starting point.

Lemma 34 Let $U \leq G$ be regular such that $U \not \leq S_{h} \times S_{n} \times\{\mathrm{id}\},\left(p^{j}\right)_{j \in \mathcal{P}^{U}} \in \mathfrak{S}(U)$ and $C \in \mathfrak{C}_{k}^{U}$. If $j \in \mathcal{P}_{2}^{U}$ is such that $\left|C\left(p^{j}\right)\right|=1$, then $A_{C}^{2}\left(p^{j}\right) \neq \varnothing$.

Proof Let $C\left(p^{j}\right)=\left\{x_{1}\right\}$ where $x_{1}$ is a $k$-subset of $N$ and assume, by contradiction, that $A_{C}^{2}\left(p^{j}\right)=\varnothing$. Thus, by (32), we have $\psi_{j} x_{1}=x_{1}$, with $\psi_{j} \in S_{n}$ defined in (30). Since $j \in \mathcal{P}_{2}^{U}$, there exists $\left(\varphi_{1}, \psi_{1}, \rho_{0}\right) \in \operatorname{Stab}_{U}\left(p^{j}\right)$ and, by the regularity of $U$, we have $\psi_{1}=\psi_{j}$. Thus, $\psi_{1} x_{1}=x_{1}$. It follows that $\psi_{1}^{-1} C\left(p^{j}\right)=C\left(p^{j}\right)$. On the other hand, since $U \not \leq S_{h} \times S_{n} \times\{\mathrm{id}\}$, there exists $\left(\varphi_{*}, \psi_{*}, \rho_{0}\right) \in U$ and, by (26) and (8), we deduce that

$$
\begin{aligned}
C\left(p^{j\left(\varphi_{*}, \psi_{*}, \rho_{0}\right)}\right) & =C\left(\left(p^{j\left(\varphi_{1}, \psi_{1}, \rho_{0}\right)}\right)^{\left(\varphi_{*} \varphi_{1}^{-1}, \psi_{*} \psi_{1}^{-1}, \mathrm{id}\right)}\right) \\
& =C\left(p^{j\left(\varphi_{*} \varphi_{1}^{-1}, \psi_{*} \psi_{1}^{-1}, \mathrm{id}\right)}\right)=\psi_{*} \psi_{1}^{-1} C\left(p^{j}\right)=\psi_{*} C\left(p^{j}\right),
\end{aligned}
$$


which contradicts (9).

Proof of the implication (ii) $\Rightarrow$ (i) of Theorem 10 Assume that one among (a)-(d) holds and let $C \in \mathfrak{C}_{k}^{U}$ be decisive. We will prove that $\mathfrak{F}_{k, C}^{U} \neq \varnothing$.

If (a) holds then, by (28), we have $\mathcal{P}_{2}^{U}=\varnothing$. Hence, by Theorem 33, we immediately deduce $\mathfrak{F}_{k, C}^{U} \neq \varnothing$. Assume then that (a) does not hold but one among (b)-(d) holds. Then, using the other implication in (28), we have $\mathcal{P}_{2}^{U} \neq \varnothing$ and, by Theorem 33, we need to show that, for every $j \in \mathcal{P}_{2}^{U}, A_{C}^{2}\left(p^{j}\right) \neq \varnothing$.

Assume by contradiction that for some $j \in \mathcal{P}_{2}^{U}$, we have $A_{C}^{2}\left(p^{j}\right)=\varnothing$. Thus (32) holds true, for $\psi_{j} \in S_{n}$ defined by(30). Recall now that $\psi_{j}$ is a conjugate of $\rho_{0}$. Thus, if $n$ is even all its orbits have size 2; if $n$ is odd we have a unique orbit of size 1 given by the only fixed point of $\psi_{j}$ and all the other orbits have size 2 .

Assume first that $k=1$. Then, for every $x \in C\left(p^{j}\right), x$ is a singleton and the unique element in $x$ is a fixed point for $\psi_{j}$. Since, for $n$ even, $\psi_{j}$ has no fixed point we deduce that $n$ is odd and $C\left(p^{j}\right)=\left\{\left\{x_{1}\right\}\right\}$ where $x_{1}$ is the only fixed point of $\psi_{j}$. Thus, by Lemma 34, we get the contradiction $A_{C}^{2}\left(p^{j}\right) \neq \varnothing$.

Assume now that $n \leq 3$. Because of the previous step, we need to consider only the case $n=3$ and $k=2$. Thus, every $x \in C\left(p^{j}\right)$ is a 2-subset of $N$ fixed by $\psi_{j}$. But $T_{\psi_{j}}=[1,2]$, that is, $\psi_{j}=(a b)$ for some distinct $a, b \in N$. Thus, the only possibility is $x=\{a, b\}$ and hence $C\left(p^{j}\right)=\{\{a, b\}\}$ is a singleton. Again Lemma 34 gives the internal contradiction $A_{C}^{2}\left(p^{j}\right) \neq \varnothing$.

Assume next that $n$ is even and $k$ is odd. Every $x \in C\left(p^{j}\right)$ is a $k$-subset of $N$ fixed by $\psi_{j}$. But since every orbit of $\psi_{j}$ has size 2 , any subset of $N$ fixed by $\psi_{j}$ has even size. Thus $C\left(p^{j}\right)=\varnothing$ against decisiveness.

Finally, assume that $k=n-1$. If $n$ is even, then $k$ is odd and we conclude by the previous step. If $n$ is odd, we have that every $x \in C\left(p^{j}\right)$ is a $(n-1)$-subset of $N$ fixed by $\psi_{j}$. But the unique subset of $N$ of size $n-1$ fixed by $\psi_{j}$ is the union of the orbits of $\psi_{j}$ of size 2 , that is $N \backslash\left\{x_{1}\right\}$, where $x_{1}$ is the unique fixed point of $\psi_{j}$. Thus, $\left|C\left(p^{j}\right)\right|=1$ and Lemma 34 gives the contradiction $A_{C}^{2}\left(p^{j}\right) \neq \varnothing$.

We are now left with proving the implication $(i) \Rightarrow(i i)$ of Theorem 10 . To that purpose, we need to introduce and study a special family of $k$-SCCs.

Let $U \leq G$ be regular. For every $p \in \mathcal{P}$, denote by $\psi_{p}$ the unique permutation in $S_{n}$ such that

$$
\operatorname{Stab}_{U}(p) \subseteq\left(S_{h} \times\{\mathrm{id}\} \times\{\mathrm{id}\}\right) \cup\left(S_{h} \times\left\{\psi_{p}\right\} \times\left\{\rho_{0}\right\}\right)
$$

The $k$-SCC $U_{k}$ associated with $U$ is defined, for every $p \in \mathcal{P}$, by

$$
U_{k}(p)= \begin{cases}\mathbb{P}_{k}(N) & \text { if } \operatorname{Stab}_{U}(p) \leq S_{h} \times\{\mathrm{id}\} \times\{\mathrm{id}\} \\ \left\{x \in \mathbb{P}_{k}(N): \psi_{p} x=x\right\} & \text { if } \operatorname{Stab}_{U}(p) \not \leq S_{h} \times\{\mathrm{id}\} \times\{\mathrm{id}\}\end{cases}
$$

The next two propositions show some important properties of $U_{k}$.

Proposition 35 Let $U \leq G$ be regular. The following facts are equivalent: 
(i) $U_{k}$ is decisive.

(ii) One of the following condition is satisfied:

(a) $\mathcal{P}_{2}^{U}=\varnothing$;

(b) $n$ is odd;

(c) $n$ is even with $n \geq 4$ and $k$ is even.

Proof (i) $\Rightarrow$ (ii) Let $U_{k}$ be decisive, $\mathcal{P}_{2}^{U} \neq \varnothing$ and $n$ even. We show that $n \geq 4$ and that $k$ is even. Since $\mathcal{P}_{2}^{U} \neq \varnothing$, there exists $p \in \mathcal{P} \operatorname{such}_{\text {that }} \operatorname{Stab}_{U}(p) \not \leq S_{h} \times\{$ id $\} \times\{$ id $\}$ and thus there exists $\left(\varphi, \psi_{p}, \rho_{0}\right) \in \operatorname{Stab}_{U}(p)$. By $U_{k}(p) \neq \varnothing$, we deduce that there exists at least one $k$-subset $x$ of $N$ fixed by $\psi_{p}$. Since $1 \leq k \leq n-1, x$ is a proper nonempty subset of $N$ which is union of $\psi_{p}$-orbits. Since $n$ is even, we have $n / 2$ orbits all of size 2. If $n=2$, then we have just one orbit and thus the only subsets of $N$ which are union of orbits are $\varnothing$ and $N$. It follows that $n \geq 4$ and that $k=|x|$ is even.

(ii) $\Rightarrow$ (i) Let $\mathcal{Q}=\left\{p \in \mathcal{P}: \operatorname{Stab}_{U}(p) \not \leq S_{h} \times\{\right.$ id $\} \times\{$ id $\left.\}\right\}$. Since $\mathbb{P}_{k}(N) \neq \varnothing$, in order to show that $U_{k}$ is decisive, it is enough to see that for every $p \in \mathcal{Q}$, we have $\left\{x \in \mathbb{P}_{k}(N): \psi_{p} x=x\right\} \neq \varnothing$. If $\mathcal{P}_{2}^{U}=\varnothing$, this is clear because necessarily we also have $\mathcal{Q}=\varnothing$. Let $n$ be odd and pick $p \in \mathcal{Q}$. Then $\psi_{p}$ has $\frac{n-1}{2} \geq 1$ orbits of size 2 and one orbit of size 1 . Assembling some of those orbits we can surely build a $k$-subset of $N$ fixed by $\psi_{p}$, whatever $k$ is. Thus, $\left\{x \in \mathbb{P}_{k}(N): \psi_{p} x=x\right\} \neq \varnothing$. Let finally $n$ be even with $n \geq 4$ and $k$ be even. Then, we have $2 \leq k \leq n-2$. Pick $p \in \mathcal{Q}$. Then, $\psi_{p}$ has $\frac{n}{2} \geq 2$ orbits of size 2 . Assembling some of those orbits we can surely build a $k$-subset of $N$ fixed by $\psi_{p}$. Thus, $\left\{x \in \mathbb{P}_{k}(N): \psi_{p} x=x\right\} \neq \varnothing$.

In order to state the next result, let us first define the set

$$
\begin{aligned}
T= & \left\{(n, k) \in \mathbb{N}^{2}: n \leq 3\right\} \cup\left\{(n, k) \in \mathbb{N}^{2}: k \in\{1, n-1\}\right\} \\
& \cup\left\{(n, k) \in \mathbb{N}^{2}: n \text { is even, } k \text { is odd }\right\} .
\end{aligned}
$$

Proposition 36 Let $U \leq G$ be regular. Then, the following facts hold:

(i) If $(\varphi, \psi, \rho) \in U$ and $p \in \mathcal{P}$, then $U_{k}\left(p^{(\varphi, \psi, \rho)}\right)=\psi U_{k}(p)$.

(ii) If $(n, k) \notin T$ then, for every $p \in \mathcal{P},\left|U_{k}(p)\right| \geq 2$. In particular $U_{k}$ is decisive.

(iii) If $(n, k) \notin T$ then $U_{k} \in \mathfrak{C}_{k}^{U}$.

Proof (i) Let first $p$ be such that $\operatorname{Stab}_{U}(p) \leq S_{h} \times\{$ id $\} \times\{$ id $\}$. Then, also $\operatorname{Stab}_{U}\left(p^{(\varphi, \psi, \rho)}\right) \leq S_{h} \times\{\mathrm{id}\} \times\{\mathrm{id}\}$ and thus $\bar{U}_{k}\left(p^{(\varphi, \psi, \rho)}\right)=U_{k}(p)=\mathbb{P}_{k}(N)=$ $\psi \mathbb{P}_{k}(N)=\psi U_{k}(p)$.

Let next $p$ be such that $\operatorname{Stab}_{U}(p) \not \leq S_{h} \times\{\mathrm{id}\} \times\{\mathrm{id}\}$. Then also $\operatorname{Stab}_{U}\left(p^{(\varphi, \psi, \rho)}\right) \not{\leq}$ $S_{h} \times\{\mathrm{id}\} \times\{\mathrm{id}\}$. We find the link between $\psi_{p}$ and $\psi_{p^{(\varphi, \psi, \rho)}}$. Let $\left(\varphi_{1}, \psi_{p}, \rho_{0}\right) \in$ $\operatorname{Stab}_{U}(p)$. Then, using the fact that $\Omega$ is abelian, we have

$$
\begin{aligned}
\left(\varphi_{1}, \psi_{p}, \rho_{0}\right)^{(\varphi, \psi, \rho)} & =\left(\varphi_{1}^{\varphi}, \psi_{p}^{\psi}, \rho_{0}^{\rho}\right)=\left(\varphi_{1}^{\varphi}, \psi_{p}^{\psi}, \rho_{0}\right) \in \operatorname{Stab}_{U}(p)^{(\varphi, \psi, \rho)} \\
& =\operatorname{Stab}_{U}\left(p^{(\varphi, \psi, \rho)}\right) .
\end{aligned}
$$


Thus, $\psi_{p^{(\varphi, \psi, \rho)}}=\left(\psi_{p}\right)^{\psi}$. Using $\mathbb{P}_{k}(N)=\psi \mathbb{P}_{k}(N)$ and (25), it follows that

$$
\begin{aligned}
U_{k}\left(p^{(\varphi, \psi, \rho)}\right) & =\left\{x \in \mathbb{P}_{k}(N): \psi_{p^{(\varphi, \psi, \rho)}} x=x\right\} \\
& =\left\{\psi x \in \mathbb{P}_{k}(N): x \in \mathbb{P}_{k}(N), \psi \psi_{p} \psi^{-1} \psi x=\psi x\right\} \\
& =\left\{\psi x \in \mathbb{P}_{k}(N): x \in \mathbb{P}_{k}(N), \psi_{p} x=x\right\}=\psi U_{k}(p) .
\end{aligned}
$$

(ii) Let $(n, k) \notin T$. Then, we have $k \notin\{1, n-1\}$ and $n \geq 4$. Moreover, $n$ is odd or $n$ is even and $k$ is even. Thus, by Lemma $35, U_{k}$ is decisive. Fix now $p \in \mathcal{P}$. We show that $\left|U_{k}(p)\right| \geq 2$. If $U_{k}(p)=\mathbb{P}_{k}(N)$, we have $\left|U_{k}(p)\right|=\left(\begin{array}{l}n \\ k\end{array}\right) \geq n \geq 2$. If instead $U_{k}(p)=\left\{x \in \mathbb{P}_{k}(N): \psi_{p} x=x\right\}$, then $U_{k}(p)$ is made up by all the $k$-subsets of $N$ which can be formed as union of $\psi_{p}$-orbits. Since $\left|U_{k}(p)\right| \geq 1$ we have at least one of them, say $x_{1} \in U_{k}(p)$. Let first $n$ be odd. Then, $n \geq 5$ and there are $\frac{n-1}{2} \geq 2$ orbits of $\psi_{p}$ of size 2 and one orbit of size 1 . Since $k \geq 2$, there is at least one orbit of size 2 included in $x_{1}$. On the other hand, not all the orbits of size 2 are included in $x_{1}$, otherwise $k=\left|x_{1}\right|=n-1$. Now exchange one orbit of size 2 included in $x_{1}$ with one orbit of size 2 left out. This builds another $k$-subset $x_{2}$ of $N$ belonging to $U_{k}(p)$. Let next $n$ be even and $k$ be even. Here, $n \geq 4$ and we have $\frac{n}{2} \geq 2$ orbits of $\psi_{p}$ all of size 2 . Obviously, we have used at least one orbit to build $x_{1}$ but not all and we can exchange one orbit included in $x_{1}$ with one left out building another $k$-subset $x_{2}$ of $N$ belonging to $U_{k}(p)$.

(iii) By (i) we have that condition (8) for $U$-consistency is satisfied; by (ii) we also have that, trivially, condition (9) for $U$-consistency is satisfied because it is never the case to have $U_{k}(p)$ a singleton for any $p \in \mathcal{P}$. Thus, $U_{k} \in \mathfrak{C}_{k}^{U}$.

Proof of the implication (i) $\Rightarrow$ (ii) of Theorem 10 By (28), we get the desired result proving that if $\mathcal{P}_{2}^{U} \neq \varnothing$ and $(n, k) \notin T$, then $\mathfrak{F}_{k, U_{k}}^{U}=\varnothing$. Indeed, assume that $\mathcal{P}_{2}^{U} \neq \varnothing$ and $(n, k) \notin T$ and consider $U_{k}$. By Proposition 36, we have that $U_{k} \in \mathfrak{C}_{k}^{U}$. Consider $\left(p^{j}\right)_{j \in \mathcal{P}^{U}} \in \mathfrak{S}(U)$ and pick $j \in \mathcal{P}_{2}^{U}$ so that $\operatorname{Stab}_{U}\left(p^{j}\right) \not \leq S_{h} \times\{$ id $\} \times\{$ id $\}$. Then, by definition of $U_{k}$, we have

$$
U_{k}\left(p^{j}\right)=\left\{x \in \mathbb{P}_{k}(N): \psi_{p^{j}} x=x\right\}=\left\{x \in \mathbb{P}_{k}(N): \psi_{j} x=x\right\} .
$$

Therefore we surely have that, for every $x \in U_{k}(p), \psi_{j} x=x$ and (32) is satisfied, so that $A_{U_{k}}^{2}\left(p^{j}\right)=\varnothing$ and, by Theorem $33, \mathfrak{F}_{k, U_{k}}^{U}=\varnothing$.

\section{References}

Bredereck, R., Faliszewski, P., Igarashi, A., Lackner, M., Skowron, P.: Multiwinner elections with diversity constraints. In: Proceedings of the Thirty-Second AAAI Conference on Artificial Intelligence (AAAI18), pp. 933-940 (2018)

Bubboloni, D., Gori, M.: Anonymous and neutral majority rules. Soc. Choice Welf. 43, 377-401 (2014)

Bubboloni, D., Gori, M.: Symmetric majority rules. Math. Soc. Sci. 76, 73-86 (2015)

Bubboloni, D., Gori, M.: On the reversal bias of the Minimax social choice correspondence. Math. Soc. Sci. 81, 53-61 (2016a)

Bubboloni, D., Gori, M.: Resolute refinements of social choice correspondences. Math. Soc. Sci. 84, 37-49 (2016b) 
Campbell, D.E., Kelly, J.S.: Majority selection of one alternative from a binary agenda. Econ. Lett. 110, 272-273 (2011)

Campbell, D.E., Kelly, J.S.: Anonymity, monotonicity, and limited neutrality: selecting a single alternative from a binary agenda. Econ. Lett. 118, 10-12 (2013)

Campbell, D.E., Kelly, J.S.: The finer structure of resolute, neutral, and anonymous social choice correspondences. Econ. Lett. 132, 109-111 (2015)

Celis, L.E., Huang, L., Vishnoi, N.K.: Multiwinner voting with fairness constraints. In: Proceedings of the Twenty-Seventh International Joint Conference on Artificial Intelligence (IJCAI-18), pp. 144-151 (2018)

Chichilnisky, G.: Social choice and the topology of spaces of preferences. Adv. Math. 37, 165-176 (1980)

Doğan, O., Giritligil, A.E.: Anonymous and neutral social choice: existence results on resoluteness. Murat Sertel Center for Advanced Economic Studies, Working paper series 2015-01 (2015)

Eğecioğlu, Ö.: Uniform generation of anonymous and neutral preference profiles for social choice rules. Monte Carlo Methods Appl. 15, 241-255 (2009)

Eğecioğlu, Ö., Giritligil, A.E.: The impartial, anonymous, and neutral culture model: a probability model for sampling public preference structures. J. Math. Sociol. 37, 203-222 (2013)

Elkind, E., Faliszewski, P., Skowron, P., Slinko, A.: Properties of multiwinner voting rules. Soc. Choice Welf. 48, 599-632 (2017)

Faliszewski, P., Skowron, P., Slinko, A., Talmon, N.: Multiwinner voting: a new challenge for social choice theory. In: Endriss, U. (ed.) Trends in Computational Social Choice, Chapter 2, pp. 27-47. AI Access Foundation (2017) (ISBN: 978-1-326-91209-3)

Jacobson, N.: Basic Algebra I. W.H Freeman and Company, New York (1974)

Jeong, H., Ju, B.G.: Resolute majority rules. Theory Decis. 82, 31-39 (2017)

Kelly, J.S.: Symmetry groups. Soc. Choice Welf. 8, 89-95 (1991)

Lang, G., Skowron, P.: Multi-attribute proportional representation. In: Proceedings of the Thirtieth AAAI Conference on Artificial Intelligence (AAAI-16), pp. 530-536 (2016)

McMorris, F.R., Mulder, H.M., Novick, B., Powers, R.C.: Majority rule for profiles of arbitrary length, with an emphasis on the consistency axiom. Instituut voor Discrete Wiskunde Amsterdam, IDWA-Rapport 2017-4. Preprint (2020)

Moulin, H.: The strategy of social choice. In: Advanced Textbooks in Economics. North Holland Publishing Company (1983) (ISBN-10: 0444863710)

Moulin, H.: Condorcet's principle implies the no-show paradox. J. Econ. Theory 45, 53-64 (1988)

Perry, J., Powers, R.C.: Aggregation rules that satisfy anonymity and neutrality. Econ. Lett. 100, 108-110 (2008)

Powers, R.C.: Maskin monotonic aggregation rules and partial anonymity. Econ. Lett. 106, 12-14 (2010)

Quesada, A.: The majority rule with a chairman. Soc. Choice Welf. 40, 679-691 (2013)

Saari, D.G.: Geometry of Voting. Studies in Economic Theory, vol. 3. Springer, Berlin (1994)

Saari, D.G., Barney, S.: Consequences of reversing preferences. Math. Intell. 25, 17-31 (2003)

Szpilrajn, E.: Sur l'extension de l'ordre partiel. Fundam. Mat. 16, 386-389 (1930)

Publisher's Note Springer Nature remains neutral with regard to jurisdictional claims in published maps and institutional affiliations. 